Published in final edited form as:

Nat Genet. 2015 November ; 47(11): 1294-1303. doi:10.1038/ng.3412.

\title{
Large-scale genomic analyses link reproductive ageing to hypothalamic signaling, breast cancer susceptibility and BRCA1-mediated DNA repair
}

\author{
A full list of authors and affiliations appears at the end of the article. \\ \# These authors contributed equally to this work.
}

\section{Abstract}

Menopause timing has a substantial impact on infertility and risk of disease, including breast cancer, but the underlying mechanisms are poorly understood. We report a dual strategy in 70,000 women to identify common and low-frequency protein-coding variation associated with age at natural menopause (ANM). We identified 44 regions with common variants, including two harbouring additional rare missense alleles of large effect. We found enrichment of signals in/near genes involved in delayed puberty, highlighting the first molecular links between the onset and end of reproductive lifespan. Pathway analyses revealed a major association with DNA damageresponse (DDR) genes, including the first common coding variant in BRCA1 associated with any complex trait. Mendelian randomisation analyses supported a causal effect of later ANM on breast cancer risk ( $~ 6 \%$ risk increase per-year, $\mathrm{P}=3 \times 10^{-14}$ ), likely mediated by prolonged sex hormone exposure, rather than DDR mechanisms.

\section{Introduction}

Younger age at natural (non-surgical) menopause (ANM) is associated with lower risk of breast cancer, but higher risks of osteoporosis, cardiovascular disease and type 2 diabetes ${ }^{1}$. Early menopause also has a substantial impact on fertility. It is estimated that natural fertility ceases on average 10 years before menopause ${ }^{2}$, which is becoming increasingly relevant as women in many populations are delaying childbearing. For example, the birth rate in British women aged 30-34 years is now higher than in any other half decade (http:// www.ons.gov.uk/ons/publications/). ANM is on average 51 years in Caucasian populations, while natural menopause before the age of 40, or primary ovarian insufficiency (POI), occurs in $1 \%$ of the population ${ }^{3}$.

Previous genome wide association studies (GWAS) identified 18 common genetic loci associated with ANM, implicating several plausible gene candidates across a number of molecular pathways 4,5 . Together those reported variants explained $<5 \%$ of the variation in

\footnotetext{
Users may view, print, copy, and download text and data-mine the content in such documents, for the purposes of academic research, subject always to the full Conditions of use:http://www.nature.com/authors/editorial_policies/license.html\#terms

\#correspondence to John R.B. Perry (john.perry@mrc-epid.cam.ac.uk).

$\S$ These authors jointly supervised this work

There are no competing financial interests.
} 
ANM, compared to $21 \%$ explained by all common variants on GWAS arrays ${ }^{4}$. We therefore undertook a more comprehensive genetic analysis in a substantially larger sample of nearly 70,000 women, incorporating both common and, for the first time, low-frequency coding variants. We were able to triple the number of independent signals associated with ANM, including two low frequency coding variants in previously unreported loci. Our findings provide new insights into the causal relationship between ANM with breast cancer and identify molecular overlaps between ANM and puberty timing.

\section{Results}

\section{GWAS HapMap 2 meta-analysis}

In a combined analysis of up to 69,360 women of European ancestry (Supplementary Table 1), 1,208 SNPs, among a total of $\sim 2.6$ million, reached the genome-wide significance threshold $\left(\mathrm{P}<5 \times 10^{-8}\right)$ for association with ANM. Of these, we identified 54 independent signals located in 44 genomic regions using approximate conditional analysis implemented in GCTA (Figure 1, Table 1, Supplementary Tables 2 and 3). Eight loci contained secondary signals: six loci each contained two signals, and two loci each contained three signals. Across the 54 identified signals, MAFs ranged from 7\% to 49\%, and effect sizes from 0.07 to 0.88 years per allele with no significant heterogeneity between studies. All of the 18 previously reported independent signals for $\mathrm{ANM}^{4,5}$ retained directionally concordant genome-wide significance (maximum $\mathrm{P}=3.7 \times 10^{-11}$ ). These 18 signals were also directionally concordant in a sub-meta-analysis of studies that were not included in the previous publication (P-value range $1 \times 10^{-30}$ to $1 \times 10^{-3}$ ). The top 29,958 independent SNPs with association $\mathrm{P}<0.05$ explained $21 \%$ (SE 9.7\%, $\mathrm{P}=0.01$ ) of the variance in ANM reducing to $6 \%$ ( $\mathrm{SE} 1.6 \%, \mathrm{P}=6.3 \times 10^{-12}$ ) for the top $54 \mathrm{SNPs}$ with $\mathrm{P}<5 \times 10^{-8}$ (Supplementary Table 4). This contrasts with an estimate of $2.6 \%$ for the previously identified 18 index SNPs.

We assessed functional enrichment of all ANM-SNP associations in regions containing active histone marks across 10 physiological cell-type groups using stratified LD score regression ${ }^{6}$ (see Methods and Supplementary Table 5). Only the 'kidney related cell types' group showed significant enrichment $(\mathrm{P}=0.003)$, which could reflect the mesonephric embryonic origin of ovarian parenchymal cells ${ }^{7}$. Analysis by functional annotation revealed the strongest enrichment for variants located in UCSC defined coding regions (Supplementary Table 5), with $\sim 1.5 \%$ of SNPs explaining $24.8 \%$ of the trait heritability $\left(\mathrm{P}=4.6 \times 10^{-3}\right)$. The heritable component increased to $55 \%$ (SE $\left.11 \%, \mathrm{P}=2.9 \times 10^{-7}\right)$ when a $500 \mathrm{bp}$ window was added to the coding regions, capturing $\sim 6.5 \%$ of SNPs.

\section{Exome array meta-analysis}

To estimate the contribution of low-frequency coding variation to ANM, we performed a meta-analysis of up to 39,026 women genotyped on exome arrays (Supplementary Table 6). Only one signal, from two highly correlated $\left(\mathrm{r}^{2}=0.73, \mathrm{D}^{\prime}=1\right)$ low-frequency missense variants in $H E L B$, reached genome-wide significance in this discovery phase (Table 2, Figure 1, Supplementary Table 7). Ten low-frequency (MAF<5\%), non-synonymous SNPs with association $\mathrm{P}<5 \times 10^{-4}$ were selected for follow-up in an independent sample of 10,157 women from the deCODE study that imputed rare variant genotypes. Directionally 
concordant effect estimates were observed for $6 / 8$ variants ( 2 of the 10 failed QC). The combined analysis identified missense alleles in $H E L B$ (rs75770066, MAF $=3.6 \%$, beta $=$ 0.85 year/allele, $\mathrm{P}=1.2 \times 10^{-31}$ ) and SLCO4A1 (rs140267842, MAF $=0.8 \%$, beta $=0.79$, $\mathrm{P}=1.6 \times 10^{-8}$ ) as associated with ANM (Table 2, Supplementary Table 7 and Supplementary Figure 1).

HELB is a DNA helicase that unwinds DNA during replication, transcription, repair and recombination. SLCO4A1 (solute carrier organic anion transporter family, member 4A1) transports organic anions such as thyroid hormones and estrone-3-sulfate. Both exome array signals in $H E L B$ and $S L C O 4 A 1$ were located in ANM loci newly identified by our parallel HapMap2 GWAS meta-analysis. At HELB the association of the common index SNP, rs12371165, was fully explained by associations at the two rare exome chip SNPs, which are in high LD with each other $\left(\mathrm{r}^{2}=0.73, \mathrm{D}^{\prime}=1\right)$ (Figure 2$)$. In contrast, the three independent signal SNPs identified through GCTA were not explained by the rare variant(s) (Supplementary Table 8). It thus appears there are at least two non-redundant signals at this locus and future fine-mapping experiments will be required to fully elucidate the number of independent causal variants. Functional studies have shown that substitution of aspartate by a non-polar residue at amino acid 506 of $H E L B$ affects binding of HELB to Replication Protein A (RPA) ${ }^{8}$. At $S L C 04 A 1$, all three variants (the common index SNP, second signal from GCTA and the exomechip variant) appeared to reflect non-redundant signals, such that the association of each with ANM was unaffected by the presence of either of the others (Supplementary Table 8).

\section{ANM SNPs strongly enriched in DNA damage-response pathways}

Pathway analyses using MAGENTA and GRAIL indicated substantial enrichment of GWAS SNP associations in DNA damage response (DDR) pathways (Supplementary Tables 9 and 10). Seven of the 10 ANM pathways identified by MAGENTA at study-wise significance were involved in DDR, with the highest enrichment in the PANTHER defined 'DNA Repair Pathway' $\left(\mathrm{P}=1 \times 10^{-6}\right)$. After annotating likely causal genes at each locus, we found that 29 of the 44 GWAS highlighted regions contained one or more DDR genes within 500kb (Table 1). At 18 of these 29 regions, the DDR candidate was either the nearest gene or the signal was associated with expression of a DDR gene at the locus.

The top SNP at GWAS Signal \#37 (Table 1 ) was highly correlated $\left(\mathrm{r}^{2}>0.95\right)$ with four common non-synonymous variants in BRCA1 [rs1799966, rs16942, rs 16941, rs799917], none of which is listed in HGMD (wWw.hgmd.cf.ac.uk/) as a known breast cancer susceptibility variant and all of which are listed as "not clinically important" on the Breast Information Core http://research.nhgri.nih.gov/bic/. In our exome array data, no low frequency coding variants in $B R C A 1$ were associated with ANM ( $\mathrm{P}>0.05)$. Signal \#37 was an eQTL for BRCA1 in multiple tissues, including: blood, skin, adipose and brain (Supplementary Table 11). There were 15 ANM signal genes that STRING analysis identified as having at least one direct link to BRCA1 (Supplementary Table 12, Supplementary Figure 2). Of these, there is experimental evidence that 7 code for direct binding partners of BRCA1: BRE (Signal \#5), MSH6 (Signal \#6), POLR2H (Signal \#8), 
FAM175A (Signal \#9), UIMC1 (Signal \#13), RAD51 (Signal \#30), and CHEK2 (Signal \#43).

While many of the DDR genes highlighted are involved in homologous recombination for repair of double strand breaks, such as the BRCA1 pathway, other mechanisms of repair are also represented, eg. mismatch repair (MSH5, MSHO) and base excision repair ( $A P E X 1$, PARP2) (Figure 3). Two genes act as DNA damage checkpoints (CHEK2 and BRSK1), others are involved in the cellular response to damage, such as cell cycle arrest, DNA replication, transcription control and apoptosis (Figure 3). CHEK2 is a well-known breast cancer associated gene ${ }^{9}$, but the ANM-associated signal was not in LD with the 1100delC variant associated with breast cancer $\left(r^{2}<0.01\right)$.

\section{ANM SNPs enriched in known POI genes}

In addition to the DDR pathways, MAGENTA analyses also identified a four-fold enrichment of ANM GWAS SNP associations located in/near a set of 31 genes reportedly associated with monogenic primary ovarian insufficiency (Supplementary Tables $13 \& 14$ ). Four of our genome-wide significant hits were located in or near reported POI genes. Autosomal recessive mutations in $M C M 8$ cause primary amenorrhea, hypothyroidism, and hypergonadotropic hypogonadism ${ }^{10}$. Recessive mutations in EIF2B4 (signal \#5) cause ovarioleukodystrophy with vanishing white matter syndrome ${ }^{11}$. POLG (signal \#31) mutations have been linked to POI in isolation or associated with other neurologic conditions ${ }^{12}$. Mutations in MSH5 (Signals \#15a and \#15b) have been associated with various human diseases including POI ${ }^{13}$. In addition, TDRD3 (Signal \#28) is a primary binding partner of $F M R 1$ in which triplet repeat premutations are a risk factor for $\mathrm{POI}{ }^{14}$. We saw no significant enrichment of ANM signals in our wider panel of ovarian function genes (Supplementary Tables 13 and 15).

\section{Genetic correlation of ANM with other traits/diseases}

We searched the GRASP database ${ }^{15}$ and NHGRI catalogue (http://www.genome.gov/ gwastudies/) for pleiotropy between ANM signals and proxies (r2>0.5) with other GWAS traits (Supplementary Table 16). The top overlapping signals were for liver enzymes, lipids, urate, height and fasting glucose $\left(\mathrm{p}=<10^{-10}\right.$ for association of ANM SNP/proxy and second trait). We found no overlap with any autoimmune traits and only a very weak link with any cancer (upper airway tract cancer, $\mathrm{p}=1 \times 10^{-8}$ ). To test the relationship between ANM and other health outcomes more broadly, we performed cross-trait LD score regression to estimate genetic correlation with 53 published GWAS meta-analyses (Supplementary Table 17). Adult obesity ranked highest in this analysis with a negative trait correlation $\left(r_{g}=-0.15\right.$, $\mathrm{P}=0.0004)$ with supporting evidence from other growth/anthropometric traits including age at menarche $\left(\mathrm{r}_{\mathrm{g}}=0.14, \mathrm{P}=0.003\right), \mathrm{BMI}\left(\mathrm{r}_{\mathrm{g}}=-0.13, \mathrm{P}=0.003\right)$, $\mathrm{BMI}$ in women but not men $(\mathrm{P}=0.002$ vs 0.17$)$, waist circumference in women but not men ( $\mathrm{P}=0.009$ vs 0.29$)$ and WHR in men but not women ( $\mathrm{P}=0.03$ vs 0.27 ). Other nominally significant associations include HDL $\left(\mathrm{r}_{\mathrm{g}}=0.14, \mathrm{P}=0.02\right)$ and current/former smoking status $\left(\mathrm{r}_{\mathrm{g}}=0.20, \mathrm{P}=0.04\right)$ both of which are supported by epidemiological observations ${ }^{16}$. 
To elucidate the causal directions between these traits, we performed bi-directional Mendelian randomisation (MR) analyses on ANM with both age at menarche and BMI. We were unable to resolve the causal direction with BMI (BMI to ANM: $\mathrm{P}_{\text {score }}=0.668$ (Supplementary Table 18); ANM to BMI: $P_{\text {Binomial }}=0.683$, (Supplementary Table 19). However the 123 reported menarche SNPs collectively predicted ANM in the expected direction $\left(\mathrm{P}_{\text {score }}=0.0005\right.$, Supplementary Table 20$)$, but the ANM SNP score was not associated with age at menarche $\left(\mathrm{P}_{\text {score }}=0.571\right.$, Supplementary Table 21$)$. We further explored the nature of this shared genetic architecture by testing for enrichment of all ANMassociated SNPs in/near genes implicated in monogenic or polygenic puberty timing ${ }^{17}$. Significant enrichment was found with the monogenic set $(\mathrm{P}=0.01)$, underscored by ANMassociated SNPs in/near five genes reportedly causal for hypogonadotrophic hypogonadism (KISS1R, TAC3, CHD7, SOX10 and FGFR1) (Supplementary Table 22).

\section{ANM variants demonstrate causal link with breast cancer}

Given the overwhelming enrichment of DDR genes and known epidemiological associations between ANM and breast cancer risk ${ }^{18}$, we tested the causal relationships between these traits using a Mendelian Randomization approach ${ }^{19}$.

Across the 56 ANM SNPs (54 HapMap $2+2$ exome) there was a positive correlation between the effect sizes on ANM and the effect sizes for risk (logORs) of breast cancer (in 46,347 breast cancer cases and 41,736 controls from Breast Cancer Association Consortium (BCAC); $r=0.67, P=2.25 \times 10^{-8}$ ). A polygenic risk score comprising numbers of ANMincreasing alleles at the 56 SNPs, weighted by the effect size on ANM, was positively associated with breast cancer risk; each one-year older genetically predicted ANM was associated with a $\mathrm{OR}=1.064$ higher breast cancer risk (1.050-1.081), $\mathrm{P}=2.78 \times 10^{-14}$ (Supplementary Figure 3). This effect size is larger than that reported by the largest pooled analysis of observational epidemiological studies $(\mathrm{OR}=1.030(1.026-1.034))^{18}$. All of the women in the GWAS from the BCAC study were also included in the Mendelian randomization (MR) study ( $\mathrm{N}=14884, \sim 14 \%$ of total MR study). To confirm that this overlap did not bias our results we conducted two analyses. Firstly, a sensitivity analysis tested the effect on breast cancer of 18 previously identified ANM SNPs, which were identified from a meta-analysis that did not include BCAC cases, and a similar effect estimate was observed (OR 1.062 [1.033-1.101, P=1.58 $\left.\times 10^{-7}\right]$ ) Secondly, the reverse analysis tested 63 SNPs with independent robust associations with breast cancer ${ }^{20}$, and found no association between these breast cancer signals and ANM $\left(\mathrm{P}_{\text {score }}>0.05\right)$, which reduces the likelihood of caseascertainment bias in our discovery meta-analysis (Supplementary Table 23).

Stratified analyses revealed significantly larger effect estimates for the ANM risk score in ER positive vs ER negative breast cancer cases $\left(\mathrm{OR}=1.07(1.05-1.10) \mathrm{P}=1.73 \times 10^{-12}\right.$ vs $\mathrm{OR}=1.03(1.00-1.07) \mathrm{P}=0.043 ; \mathrm{P}=0.0086$ for the case-only analysis) and women aged $>=55$ vs $<=45$ years $(\mathrm{OR}=1.06(1.04-1.10) \mathrm{P}=2.23 \times 10-7$ vs $\mathrm{OR}=1.00(0.97-1.05) \mathrm{P}=0.95$, caseonly $\left.\mathrm{P}=2.30 \times 10^{-5}\right)$. Consideration of DDR vs non-DDR linked SNPs in the polygenic risk score also produced discordant effect estimates (OR 1.05 [1.03-1.08], $\mathrm{p}=1.06 \times 10^{-7}$ vs OR 1.12 [1.06-1.21], $\mathrm{P}=7.84 \times 10^{-10}$ respectively, $\mathrm{P}_{\text {het }}=0.01$ ), a difference which was further reinforced in the age stratified analyses (Supplementary Figure 3 and Table 24). 
Furthermore, lack of association between ANM risk scores with risk of prostate cancer in men (in 25,074 cases and 24,272 controls) ( $\mathrm{P}=0.36$, Supplementary Table 25) provides no evidence to support an effect of ANM-related DDR mechanisms on other cancer risks. We therefore surmise that ANM genetic variants influence breast cancer risk primarily through variation in menopause timing.

\section{Discussion}

Our study represents a largely expanded genetic discovery effort for ANM, both in terms of increased sample size and breadth of variation tested. By more than doubling the GWAS sample size we have increased the number of loci robustly associated with the trait threefold. In addition, we assessed the role of low-frequency protein coding variation using exome genotyping arrays. This approach identified the first such variants of large effect for ANM, implicating both HELB and SLCO4A1 in the aetiology of reproductive ageing. Both of these regions contain common variants we identified in parallel, producing "synthetic associations" at the HELB locus ${ }^{21}$.

Our analyses suggest a far more substantial role for DNA damage response processes in ovarian ageing than originally estimated. Both manual assessment and formal computational approaches identified an overwhelming excess of DDR genes mapping to the 44 GWAS loci, possibly explaining up to $2 / 3$ rds of the associations. Despite the limitations of our GWAS approach to map definitively SNPs to genes, 19/44 loci contained signal SNPs where plausible DDR candidates were either the closest gene or linked via altered expression levels to the associated variant. This level of enrichment is comparable to that observed in GWAS meta-analyses of several cancers 22,23 .

A notable inclusion in our list of DDR annotated genes was BRCA1, which was the nearest gene, linked as an eQTL and contained multiple non-synonymous SNPs in high LD with the lead index SNP. Although rare loss of function alleles are well studied in the context of cancer pre-disposition, coding variants in $B R C A 1$ are generally regarded as neutral and have not been previously mapped to any complex trait or disease, including breast cancer. Titus et al have shown that BRCA1 expression decreases in human ovaries with age and that reduced brcal expression in mouse models leads to reduced ovarian reserve ${ }^{24}$. This is consistent with our data, where the ANM-lowering allele reduces expression in blood. BRCA1 directly inhibits a functional interaction with oestrogen receptor a and thus BRCA1 variants could also affect ANM through altered oestrogen signalling ${ }^{25}$. Of the 34 DDR genes highlighted in Table 1, 15 have experimental links to BRCA1, three of which form part of the BRCA1-A complex; BRE (BRCC45), FAM175A (Abraxas) and UIMC1 (RAP80). While dispensable for BRCA1's major tumour suppressive role in promoting DNA double-strand break repair by homologous recombination (HR), the BRCA1-A complex components RAP80 and Abraxas are actually involved in counteracting this activity, restricting BRCA1-dependent HR to appropriate levels ${ }^{26}$. Similarly, the DNA helicase Fbh1 (FBXO18; Signal \#20) negatively regulates $\mathrm{HR}^{27,28}$. While $\mathrm{HR}$ is essential for cell viability, such anti-recombinase activities are also important for maintaining genome stability, and failure of this regulation is associated with inappropriate recombination events, and the accumulation of toxic 
recombination intermediates, DNA repair activities associated with driving translocations, loss-of-heterozygosity, and chromosomal abnormalities ${ }^{29}$.

Double strand break repair is an important response to metabolic and environmental damage to DNA, but is also a key process in meiosis for resolving recombination events. Aberrant meiotic recombination is known to cause meiotic arrest and affect the viability of oocytes. Menopause occurs when the number of oocytes in the ovary falls below a threshold number (approx. 1000) and thus processes that affect the size of the oocyte pool will affect timing of menopause. Recent studies have shown that recessive mutations in both MCM8 and MCM9 results in genomic instability, caused by a deficiency in double strand break repair, which has a devastating effect on the oocyte pool, causing POI ${ }^{10,30}$. MCM8 is one of the genes highlighted in our study (signal \#41) and a further 12 are also involved in homologous recombination repair, including two which are specific for meiotic repair (MSH5 and DMC1 (DNA meiotic recombinase 1)). Thus double strand break repair, during recombination, at meiosis, appears to be a major mechanism by which oocyte numbers are regulated, thus determining depletion of the oocyte pool and ANM.

In this study, however, the repair mechanisms highlighted are not confined to homologous recombination repair; mismatch repair and base excision repair are also implicated, as well as mitotic repair and repair checkpoints. Thus it appears that the mechanisms are not confined to repair of meiotic cross-overs, but more general mechanisms are also involved. Seven million oogonia are produced during fetal development by mitosis. Inefficient repair of DNA damage during these mitotic events could result in apoptosis and thus a reduction in the initial oocyte pool. Loss of oocytes throughout female life is predominantly by atresia rather than ovulation. It is likely that oocytes are particularly sensitive to DNA damage due to the prolonged state of cell cycle arrest, lasting up to 50-60 years. Thus aberrant repair throughout life could affect the rate of atresia and thus ANM.

Several of the genes highlighted in our study are robust cancer predisposition genes, eg. BRCA1, CHEK2 and MSH6. Additionally BCAR4 and STARD3 have also been linked with breast cancer predisposition. However common susceptibility variants have not been mapped to any of these genes through GWAS approaches for any cancer [www.genome.gov/ gwastudies/]. Patients with known pathogenic BRCA1 breast cancer predisposition mutations, have been reported to have lower ANM ${ }^{31}$, although other studies have failed to replicate these findings ${ }^{32}$.

We found that carrying higher numbers of ANM-increasing variants was associated with increased breast cancer risk. This was consistent with (indeed slightly larger than) the observed epidemiological association. Our Mendelian randomization approach indicates a causal relationship between ANM and breast cancer risk, with prolonged oestrogen and/or progesterone exposure likely to be the mechanism ${ }^{33}$. Consistent with this, the effect size was greater for ER-positive than ER-negative breast cancer.

At first sight, this observation might appear paradoxical given the enrichment of DDR genes associated with menopause. However, we noted that the association between ANM variants and breast cancer risk was weaker for those in/near DDR genes than those in the non-DDR 
set. This raises the possibility that the DDR variants that reduce menopausal age do modestly increase breast cancer risk, but this is counterbalanced by the larger effect due to altered hormonal exposure. Alternatively, it is possible that variants in the non-DDR set may have a residual effect on breast cancer risk through hormonal or other mechanisms, or that both mechanisms could play a role (supplementary Figure 4). BRCA1 mutations are known to be risk factors for prostate cancer ${ }^{34}$ and yet we found no association with prostate cancer predisposition for the ANM variants, supporting the hypothesis that the breast cancer association is mediated via menopause and not a direct effect of the DDR variants. That the effect of the ANM polygenic risk score on breast cancer risk was larger than that predicted from observation studies might indicate measurement error in the reporting of age at menopause or residual negative confounding in epidemiological studies; in either case, the Mendelian Randomisation analysis performed here using the polygenic risk score as an instrumental variable can give a more accurate estimate of the effect of age at menopause on breast cancer risk. Such measurement error would also be present in studies in the ANM GWAS from which the polygenic risk score weights were derived, hence the 'true' effect of later menopause on breast cancer risk may actually be larger even than the $~ 6 \%$ increase in risk/year predicted here.

Our findings provide novel evidence for a neural influence on the timing of ovarian follicular ageing. Until now, it has been considered that hypothalamic/pituitary activity in relation to the menopause is simply secondary to the loss of feedback inhibition by ovarian hormones ${ }^{35}$. We identified five ANM loci containing genes reported causal for hypogonadotrophic hypogonadism. Of these, monogenic disruption of three (CHD7, FGFR1 and $S O X 10$ ) are causes of Kallman syndrome, characterized by anosmic hypogonadotrophic hypogonadism due to failure of embryonic migration of GNRH secreting neurons from the olfactory bulb to the hypothalamus ${ }^{36}$. In addition, KISS1R (GPR54) encodes the receptor for kisspeptin, a key hypothalamic activator of the reproductive hormone axis, and TAC3 encodes neurokinin $\mathrm{B}$, which is highly expressed in hypothalamic neurons that also express kisspeptin and promotes the pulse frequency of luteinising hormone (LH) secretion from the pituitary. A possible central influence on ovarian ageing is also supported by the ANM locus in/near $F S H B$ (which is reportedly also associated with circulating FSH levels).

Alternatively, recent studies have identified expression of TAC3, KISS1R and kisspeptin in ovarian granulosa cells ${ }^{37}$, suggesting peripheral actions of these neuropeptides and their receptors ${ }^{38}$. Indeed, GPR54-haploinsufficiency in mice leads to progressive oocyte and follicle loss without affecting gonadotropin secretion ${ }^{38}$. Regardless of their site of action, our findings indicate several mechanisms that could link the regulation of puberty to ANM, and therefore impact both the start and end of the female reproductive lifespan.

In summary, our findings suggest a surprisingly narrow range of biological pathways governing ANM, highlighting a substantial role for DNA damage response in the aetiology of ovarian ageing. We demonstrate the utility of genetics to inform epidemiological observations, revealing shared biological pathways linking puberty timing, breast cancer and reproductive ageing. 


\section{Online Methods}

\section{Menopause data collection}

ANM was self-reported and defined as the age at last naturally occurring menstrual period followed by at least 12 consecutive months of amenorrhea. Recall bias/error for ANM may have reduced our power to detect associations, but would be unlikely to introduce systematic error. We assessed this issue in our previous meta-analysis and found no significant differences in effect estimates when considering retrospective versus prospective studies ${ }^{4}$. We included women with ANM 40-60 years in our analyses, excluding those with menopause induced by hysterectomy, bilateral ovariectomy, radiation or chemotherapy, and those using hormone replacement therapy (HRT) before menopause (Supplementary Table 1). Within each of the included studies, each participant provided written informed consent and the study protocol was approved by the Institutional Review Board at the parent institution.

\section{GWAS}

A total of 33 studies contributed genome-wide association data using self-reported ANM (Supplementary Table 1). One of the 33 studies was from the Breast Cancer Association Consortium (BCAC), comprising 17 separate studies with menopause data, genotyped using an Illumina iSelect array (iCOGs) ${ }^{20}$. This resulted in a maximum total sample of 69,360 individuals of European descent. Studies were asked to use the full imputed set of HapMap Phase 2 autosomal SNPs, and to run an additive model including top principal components and study specific covariates.

In some cases, studies submitted data using 1000 Genomes based imputation; in these cases SNPs not included in the HapMap 2 set were removed. Once data were submitted, each study was quality controlled centrally according to standard QC protocols independently by two analysts. SNPs were filtered out if the minor allele frequency (MAF) was less than $1 \%$, or if the imputation quality metrics were low (imputation quality<0.4). Studies and SNPs passing QC were combined using an inverse-variance weighted meta-analysis, implemented using METAL ${ }^{39}$. Again, this meta-analysis was run by two analysts independently, who then separately used PLINK clumping commands ${ }^{40}$ to identify the most significant SNPs in associated regions (termed "Index SNPs"), using only those SNPs which had data from more than $50 \%$ of the studies. SNPs were considered genome-wide significant if $p<5 \times 10^{-8}$ ( $p$ of 0.05 Bonferroni corrected for a million tests). Comparisons were made to ensure concordance of the identified signals between the two independent analysts.

\section{Exome chip}

Exome genotyping data were analysed for 22 studies of European ancestry, with questionnaire data on ANM (Supplementary Table 6). Genotype calling was performed using the CHARGE (Cohorts for Heart and Aging Research in Genomic Epidemiology) joint calling protocol, including $X$ chromosome variants. Each contributing study carried out study-level analysis in the R-packages skatMeta or seqMeta using the skatCohort command with the top genetic principal components included in the model and alleles coded according to a common reference file (SNPInfo_HumanExome-12v1_rev5.tsv.txt from http:// 
www.chargeconsortium.com/main/exomechip) ${ }^{41}$. Following data submission, two data analysts carried out checks to ensure consistency of allele coding. We carried out a single variant meta-analysis in METAL ${ }^{39}$, giving a total sample size of 39,026, with associations considered significant if $p<5 \times 10^{-8}$. Variants were put forward for replication in the deCODE study $(\mathrm{n}=10,157)$ if they were present in more than half of studies in the discovery stage and had $p<5 \times 10^{-5}$ if MAF was less than $1 \%$ or $p<5 \times 10^{-4}$ if MAF was $1-5 \%$.

\section{Selection of independent signals / conditional analysis}

Independent signals (termed "Signal SNPs") for ANM were identified using approximate conditional analysis implemented in the GCTA software package ${ }^{42}$. Linkage disequilibrium (LD) between variants was estimated using three independently genotyped studies as reference panels - the Rotterdam Study I $(\mathrm{N}=5,974)$ and two EPIC-InterAct datasets $(\mathrm{N}=7,397$ and $\mathrm{N}=9,294)$; these comprised males and females of European ancestry with GWAS data imputed using CEU haplotypes from HapMap 2. We assumed zero correlation between SNPs more than $10 \mathrm{Mb}$ apart or on different chromosomes. We considered independent signals to be those observed by at least two of the three LD reference panels and located in a $10 \mathrm{Mb}$ region that contained a genome-wide significant SNP based on univariate test statistics.

We assessed the independence between exome array and HapMap 2 signals by performing formal conditional analyses in the Women's Genome Health Study (WGHS, N=11,664). Regression was performed including all significant index SNPs in additive models, including the same study covariates as used in the primary analysis. LD computation in Haploview ${ }^{43}$ used experimental genotypes where possible (the rare exome chip variants and the common variants rs3741604 and rs2236553), but HapMap 2 imputed genotypes for the other common variants (MaCH v. 1.0.16, all Rsq >0.99).

\section{Gene identification}

At each locus identified by the GWAS meta-analysis, we annotated the likely causative gene(s) (Supplementary Table 3) using the following criteria: identified in at least one of the gene prioritisation/pathway programs (GRAIL or STRING), the top SNP or a proxy $\left(\mathrm{r}^{2}>0.8\right)$ was an eQTL in one of 108 tissues, the top SNP or a proxy $\left(\mathrm{r}^{2}>0.8\right)$ was a coding variant (Supplementary tables 9-12, 26, 27, Supplementary Figure 5). In case of overlap between the results of the GWAS and exome analyses, the gene indicated by the exome array analysis was chosen. Further manual annotation was used to select additional likely candidates based on known biology (e.g monogenic primary ovarian insufficiency) or biology highlighted by hypothesis-free pathway testing (Supplementary Table 15). If no candidate was identified by these methods the nearest gene was chosen.

GRAIL is a literature based text mining program used to suggest the mostly likely casual gene at each locus ${ }^{44}$, controlling for gene size and without any seed regions. A GRAIL pvalue $<0.05$ was taken to indicate a suggested causal gene (Supplementary Table 9). All genes located within $500 \mathrm{~kb}$ of the top SNP at each locus were assessed using the STRING program (http://string-db.org/), which was used to highlight any connectivity between genes in different regions (Supplementary Table 12). 


\section{Expression quantitative trait loci (eQTL)}

Each independent SNP signal was assessed in over 100 separate eQTL datasets

(Supplementary methods and Table 11 for details ${ }^{45}$ ). If an independent signal SNP was in high LD ( $\mathrm{r}^{2}>0.8$; using SNAP http://www.broadinstitute.org/mpg/snap/) with the most significant signal for an eQTL, then the eQTL gene was highlighted as a potential causal candidate. The collected eQTL results met criteria for statistical thresholds for association with gene transcript levels as described in the original papers.

\section{Pathway identification}

We tested for signal enrichment across 2,580 pre-defined biological pathways in GO, KEGG, Ingenuity, Panther, Reactome and Biocarta using MAGENTA ${ }^{46}$ using the full HapMap Phase 2 imputed meta-analysis (Supplementary Table 10). Analysis was performed using the same default settings as described in our previous paper ${ }^{4}$, with study-wise significance declared at an FDR $<0.05$. In addition to these pre-defined pathways, we also tested four custom pathways comprised of genes involved in POI $(\mathrm{N}=31)$, ovarian function $(\mathrm{N}=130)$, monogenic disorders of puberty $(\mathrm{N}=21)$ and age at menarche $(\mathrm{N}=154)$

(Supplementary Tables 13-15, 22).

\section{Estimating variance explained by SNP sets}

An estimate of the total variance explained by highlighted ANM SNPs was calculated using REML (restricted maximum likelihood) implemented in GCTA ${ }^{42}$. Using individual level data from the EPIC-InterAct cohort $(\mathrm{N}=1,761)$, we calculated the attributable variance for the genome-wide significant SNPs and at varying significance thresholds $\left(5 \times 10^{-7}, 5 \times\right.$ $10^{-6}, 5 \times 10^{-5}, 5 \times 10^{-4}, 0.005,0.05$, and all SNPs passing QC) obtained from a repeated meta-analysis excluding EPIC-InterAct.

We used stratified LD score regression to quantify evidence of functional enrichment specific to groups of cell types ${ }^{6}$. We used the same baseline model as in Finucane et al. ${ }^{6}$ which comprises 53 overlapping categories including basic annotations such as coding, UTR, promoter, and intron, as well as several histone marks, DNase I Hypersensitivity Site (DHS) regions, chromHMM predictions ${ }^{47}$, regions that are conserved in mammals ${ }^{48}$, super enhancers ${ }^{49}$, and FANTOM5 enhancers ${ }^{50}$. We evaluated enrichments for each of these noncell-type specific categories. We then took 230 cell-type-specific annotations in four histone marks-H3K4me1, H3K4me3, H3K9ac ${ }^{51}$ and H3K27ac ${ }^{52}$ (Supplementary Table 5), and grouped them into 10 cell-type groups, (adrenal/pancreas; central nervous system; cardiovascular; connective/bone; gastrointestinal; immune/hematopoietic; kidney; liver; skeletal muscle; other) ${ }^{6}$. We added each cell-type group to the baseline model one at a time and measured the p-value of the resulting LD Score regression coefficient of the cell-type group using the -h2 flag in ldsc (https://github.com/bulik/ldsc) with LD Scores from 1000G Genomes Europeans [http://www.1000genomes.org/]. We ranked the cell-type groups by whether the per-SNP heritability in the 'functional' annotation was larger than the per-SNP heritability outside this annotation, controlling for the other annotations in the baseline model. 


\section{Breast and prostate cancer Mendelian Randomisation (MR)}

To assess the association of the ANM SNPs with breast cancer risk, we used breast cancer cases $(n=46,347)$ and controls $(n=41,736)$ of European ancestry from 41 studies in the BCAC, who had been genotyped using a custom Illumina Infinium array (iCOGS). Following standard quality control exclusions (as described in ${ }^{20}$ ) genotypes were available for 199,961 SNPs. Further genotypes were imputed in a two-stage procedure using SHAPEIT and IMPUTEv2 ${ }^{53}$ with the 1000 Genomes Project March 2012 release as the reference dataset ${ }^{54}$, giving $\sim 11.6$ million SNPs with imputation $\mathrm{r}^{2}>0.3$ and MAF $>0.005$. The 4,747 breast cancer cases and 7,285 controls in the BCAC dataset for whom ANM information was available had also been included in the ANM GWAS analysis.

The genotypes or imputed genotype dosages for the 56 significant SNPs in Tables 1 and 2 were used to construct a polygenic risk score for each breast cancer case and control, such that for the $\mathrm{i}^{\text {th }}$ woman

$$
P R S_{i}=\sum_{j=1}^{56} \beta_{j} G_{i j}
$$

where $\beta_{\mathrm{j}}$ is the ANM regression coefficient for the effect allele of the $\mathrm{j}^{\text {th }} \mathrm{SNP}$ (conditional $\beta \mathrm{s}$ were used for the correlated SNPs) and $\mathrm{G}_{\mathrm{ij}}$ is the number of copies of the effect allele at the $\mathrm{j}^{\text {th }} \mathrm{SNP}$ carried by the $\mathrm{i}^{\text {th }}$ woman $\left(\mathrm{G}_{\mathrm{ij}}\right.$ is between 0 and 2$)$.

The association between the polygenic risk score and breast cancer was tested using unconditional logistic regression, adjusting for study and for seven principal components (as estimated based on a subset of 37,000 uncorrelated markers including 1000 selected as ancestry informative markers). The $\log (\mathrm{OR})$ was scaled according to the effect size of a oneunit increase in polygenic risk score on ANM in control subjects, so as to obtain an estimated $\log$ OR for a one-year increase in genetically predicted ANM. Hence the polygenic risk score can be thought of as an instrumental variable in a Mendelian Randomisation of ANM against breast cancer.

Additional analyses were conducted specifically for estrogen receptor (ER) positive $(\mathrm{N}=27,026)$ or ER negative $(\mathrm{N}=7,401)$ cases, and for participants with age at diagnosis (for cases) or interview (for controls) $\leq 45$ years $(8,547$ cases and 8,029 controls) or $\geq 55$ years $(24,841$ cases and 20,410 controls)(as a surrogate for pre- or post-menopausal age at diagnosis, because ANM was not known for all participants), with heterogeneity evaluated in case-only analyses.

We also tested the association of ANM SNPs on prostate cancer risk, to determine whether any effect of genetic variants was specific to breast cancer. Prostate cancer data were available from a similar sample size to breast cancer and there is known overlap in genetic risk for breast and prostate cancer. Individual level data was not available for prostate cancer, we therefore assessed the impact of ANM using an approximated allele score comprised of the 54 HapMap2 GWAS SNPs on summary level results ${ }^{55}$. The score was assessed using summary statistics from a recent prostate cancer meta-analysis, comprising 25,074 cases and 
24,272 controls from 32 studies in the PRACTICAL Consortium ${ }^{56}$, genotyped using the iCOGs array, with quality control and imputation carried out in the same way as for the BCAC iCOGs study.

\section{Genetic correlation with additional traits}

Cross-trait LD score regression was used to estimate the genetic correlation between menopause timing and 54 individual traits from published studies including anthropometric and metabolic traits ${ }^{57}$. We estimated genetic correlations with the method described in 58 and the --rg flag in the ldsc software package (https://github.com/bulik/ldsc) with LD Scores from 1000 Genomes Europeans and default settings. Briefly, this method regresses the product of effect size estimates for trait 1 and trait 2 for each SNP against LD Score. The product of the slope and a constant estimates the genetic correlation, and the intercept estimates the product of the number of overlapping samples and the correlation between phenotypes among the overlapping samples.

Bi-directional Mendelian randomisation analyses on ANM with age at menarche and BMI were carried out using similar methods as for prostate cancer, with a weighted allele score 55 generated from summary statistics. Information on the associations with age at menarche came from the most recent genome-wide association study for the trait $(\mathrm{N}=182,416$ women from 57 studies) ${ }^{17}$. The BMI data were taken from the most recent analysis $(\mathrm{N}=249,796$ from 64 studies) ${ }^{59}$. While it was possible to calculate a full allele score for the genome-wide significant BMI SNPs to ANM analysis, this was not possible for the ANM SNPs to BMI analysis; instead a binomial test of consistency of effect direction was used.

\section{Supplementary Material}

Refer to Web version on PubMed Central for supplementary material.

\section{Authors}

Felix R. Day\#1, Katherine S. Ruth\#2, Deborah J. Thompson\#3, Kathryn L. Lunetta ${ }^{4,5}$, Natalia Pervjakova ${ }^{6,7}$, Daniel I. Chasman ${ }^{8,9}$, Lisette Stolk ${ }^{10,11}$, Hilary K. Finucane ${ }^{12,13}$, Patrick Sulem ${ }^{14}$, Brendan Bulik-Sullivan 15,16,17, Tõnu Esko6,18,19,20, Andrew D. Johnson ${ }^{5}$, Cathy E. Elks ${ }^{1}$, Nora Franceschini21, Chunyan $\mathrm{He}^{22,23}$, Elisabeth Altmaier24,25,26, Jennifer A. Brody 27 , Lude L. Franke 28 , Jennifer E. Huffman $^{5,29}$, Margaux F. Keller ${ }^{30}$, Patrick F. McArdle ${ }^{31}$, Teresa Nutile ${ }^{32}$, Eleonora Porcu ${ }^{33,34,35}$, Antonietta Robino ${ }^{36}$, Lynda M. Rose ${ }^{8}$, Ursula M. Schick ${ }^{37}$, Jennifer A. Smith $^{38}$, Alexander Teumer ${ }^{39}$, Michela Traglia ${ }^{40}$, Dragana Vuckovic ${ }^{36,41}$, Jie $\mathrm{Yao}^{42}$, Wei Zhao ${ }^{38}$, Eva Albrecht ${ }^{25}$, Najaf Amin $^{43}$, Tanguy Corre ${ }^{44,45}$, Jouke-Jan Hottenga ${ }^{46}$, Massimo Mangino ${ }^{47,48}$, Albert V. Smith ${ }^{49,50}$, Toshiko Tanaka ${ }^{51}$, Goncalo Abecasis ${ }^{35}$, Irene L. Andrulis ${ }^{52,53}$, Hoda Anton-Culver ${ }^{54}$, Antonis C. Antoniou $^{3}$, Volker Arndt ${ }^{55}$, Alice M. Arnold ${ }^{56}$, Caterina Barbieri ${ }^{36,40}$, Matthias W. Beckmann $^{57}$, Alicia Beeghly-Fadiel ${ }^{58}$, Javier Benitez ${ }^{59,60}$, Leslie Bernstein ${ }^{61}$, Suzette J. Bielinski ${ }^{62}$, Carl Blomqvist ${ }^{63}$, Eric Boerwinkle ${ }^{64,65}$, Natalia V. Bogdanova $^{66}$, Stig E. Bojesen ${ }^{67,68}$, Manjeet K. Bolla ${ }^{3}$, Anne-Lise BorresenDale 69,70 , Thibaud S Boutin ${ }^{29}$, Hiltrud Brauch ${ }^{71,72,73}$, Hermann Brenner $55,73,74$, 
Thomas Brüning ${ }^{75}$, Barbara Burwinkel ${ }^{76,77}$, Archie Campbell ${ }^{78}$, Harry Campbell ${ }^{79}$, Stephen J. Chanock ${ }^{80}$, J. Ross Chapman ${ }^{81}$, Yii-Der Ida Chen ${ }^{42}$, Georgia ChenevixTrench $^{82}$, Fergus J. Couch ${ }^{83}$, Andrea D. Coviello ${ }^{84}$, Angela Cox ${ }^{85}$, Kamila Czene ${ }^{86}$, Hatef Darabi ${ }^{86}$, Immaculata De Vivo ${ }^{12,87}$, Ellen W. Demerath ${ }^{88}$, Joe Dennis ${ }^{3}$, Peter Devilee $^{89,90}$, Thilo Dörk ${ }^{91}$, Isabel dos-Santos-Silva ${ }^{92}$, Alison M. Dunning ${ }^{93}$, John D. Eicher $^{5}$, Peter A. Fasching ${ }^{57,94}$, Jessica D. Faul ${ }^{95}$, Jonine Figueroa ${ }^{96}$, Dieter Flesch-Janys $^{97,98}$, Ilaria Gandin ${ }^{36,41}$, Melissa E. Garcia ${ }^{99}$, Montserrat GarcíaClosas $^{100,101}$, Graham G. Giles ${ }^{102,103}$, Giorgia G. Girotto ${ }^{41}$, Mark S. Goldberg ${ }^{104,105}$, Anna González-Neira ${ }^{59}$, Mark O. Goodarzi ${ }^{106}$, Megan L. Grove ${ }^{64}$, Daniel F. Gudbjartsson ${ }^{14,107}$, Pascal Guénel ${ }^{108,109}$, Xiuqing Guo ${ }^{42}$, Christopher A. Haiman $^{110}$, Per Hall ${ }^{86}$, Ute Hamann ${ }^{111}$, Brian E. Henderson ${ }^{110}$, Lynne J. Hocking ${ }^{112}$, Albert Hofman ${ }^{43}$, Georg Homuth ${ }^{113}$, Maartje J. Hooning ${ }^{114}$, John L. Hopper $^{102}$, Frank B. Hu12,87,115, Jinyan Huang ${ }^{116}$, Keith Humphreys ${ }^{86}$, David J. Hunter ${ }^{12,20,87,115}$, Anna Jakubowska117, Samuel E. Jones², Maria Kabisch ${ }^{111}$, David Karasik ${ }^{9,118}$, Julia A. Knight ${ }^{119,120}$, Ivana Kolcic ${ }^{121}$, Charles Kooperberg ${ }^{37}$, Veli-Matti Kosma122,123,124, Jennifer Kriebel 24,26,125, Vessela Kristensen 69,70,126, Diether Lambrechts ${ }^{127,128}$, Claudia Langenberg ${ }^{1}$, Jingmei Li86, Xin Li' ${ }^{12}$, Sara Lindström $^{12}$, Yongmei Liu ${ }^{129}$, Jian'an Luan ${ }^{1}$, Jan Lubinski ${ }^{117}$, Reedik Mägi' ${ }^{6}$, Arto Mannermaa ${ }^{122,123,124}$, Judith Manz ${ }^{24,26}$, Sara Margolin ${ }^{130}$, Jonathan Marten ${ }^{29}$, Nicholas G. Martin ${ }^{131}$, Corrado Masciullo ${ }^{40}$, Alfons Meindl ${ }^{132}$, Kyriaki Michailidou ${ }^{3}$, Evelin Mihailov6, Lili Milani ${ }^{6}$, Roger L. Milne ${ }^{102,103}$, Martina Müller-

Nurasyid $^{25,133,134}$, Michael Nalls ${ }^{135}$, Ben M. Neale ${ }^{15,16,17}$, Heli Nevanlinna ${ }^{136}$, Patrick Neven ${ }^{137}$, Anne B. Newman ${ }^{138,139,140}$, Børge G. Nordestgaard ${ }^{67,68}$, Janet E. Olson ${ }^{62}$, Sandosh Padmanabhan ${ }^{141}$, Paolo Peterlongo ${ }^{142}$, Ulrike Peters ${ }^{37}$, Astrid Petersmann ${ }^{143}$, Julian Peto ${ }^{92}$, Paul D.P. Pharoah ${ }^{3,93}$, Nicola N. Pirastu ${ }^{36,41}$, Ailith Pirie $^{3}$, Giorgio Pistis ${ }^{33,34,35}$, Ozren Polasek ${ }^{121}$, David Porteous ${ }^{78}$, Bruce M. Psaty27,144,145,146, Katri Pylkäs 147,148, Paolo Radice ${ }^{149}$, Leslie J. Raffel ${ }^{150,151,}$ Fernando Rivadeneira ${ }^{10,11,43}$, Igor Rudan ${ }^{79}$, Anja Rudolph ${ }^{152}$, Daniela Ruggiero ${ }^{32}$, Cinzia F. Sala ${ }^{40}$, Serena Sanna ${ }^{33}$, Elinor J. Sawyer ${ }^{153}$, David Schlessinger ${ }^{154}$, Marjanka K. Schmidt ${ }^{155}$, Frank Schmidt ${ }^{113}$, Rita K. Schmutzler ${ }^{156,157,158}$, Minouk J. Schoemaker ${ }^{100}$, Robert A. Scott ${ }^{1}$, Caroline M. Seynaeve ${ }^{114}$, Jacques Simard ${ }^{159}$, Rossella Sorice ${ }^{32}$, Melissa C. Southey ${ }^{160}$, Doris Stöck| ${ }^{26}$, Konstantin Strauch ${ }^{25,161}$, Anthony Swerdlow ${ }^{100,162}$, Kent D. Taylor ${ }^{42}$, Unnur Thorsteinsdottir ${ }^{14,50}$, Amanda E. Toland $^{163}$, Ian Tomlinson ${ }^{81,164}$, Thérèse Truong ${ }^{108,109}$, Laufey Tryggvadottir ${ }^{165}$, Stephen T. Turner ${ }^{166}$, Diego Vozzi ${ }^{36}$, Qin Wang ${ }^{3}$, Melissa Wellons ${ }^{167}$, Gonneke Willemsen ${ }^{46}$, James F. Wilson ${ }^{29,79}$, Robert Winqvist ${ }^{147,148}$, Bruce B.H.R. Wolffenbuttel $^{168,169}$, Alan F. Wright ${ }^{29}$, Drakoulis Yannoukakos ${ }^{170}$, Tatijana Zemunik $^{121}$, Wei Zheng ${ }^{58}$, Marek Zygmunt ${ }^{171}$, Sven Bergmann ${ }^{44,45}$, Dorret I. Boomsma $^{46}$, Julie E. Buring ${ }^{8,9}$, Luigi Ferrucci ${ }^{51}$, Grant W. Montgomery ${ }^{131}$, Vilmundur Gudnason ${ }^{49,50}$, Tim D. Spector ${ }^{47}$, Cornelia M van Duijn ${ }^{43}$, Behrooz Z. Alizadeh $^{172}$, Marina Ciullo ${ }^{32}$, Laura Crisponi ${ }^{33}$, Douglas F. Easton ${ }^{3,93}$, Paolo P. Gasparini 36,41 , Christian Gieger $24,25,26$, Tamara B. Harris ${ }^{99}$, Caroline Hayward ${ }^{29}$, Sharon L.R. Kardia ${ }^{38}$, Peter Kraft ${ }^{12,173}$, Barbara McKnight ${ }^{56}$, Andres Metspalu ${ }^{6}$, Alanna C. Morrison ${ }^{64}$, Alex P. Reiner ${ }^{37,144}$, Paul M. Ridker ${ }^{8,9}$, Jerome I. Rotter ${ }^{42}$, Daniela Toniolo ${ }^{40}$, André G. Uitterlinden ${ }^{10,11,43}$, Sheila Ulivi ${ }^{36}$, Henry Völzke ${ }^{39}$, 
Nicholas J. Wareham ${ }^{1}$, David R. Weir ${ }^{95}$, Laura M. Yerges-Armstrong ${ }^{31}$, PRACTICAL consortium, kConFab Investigators ${ }^{174}$, AOCS Investigators ${ }^{174}$, Generation Scotland ${ }^{175}$, EPIC-InterAct Consortium, LifeLines Cohort Study, Alkes L. Price ${ }^{12}$, Kari Stefansson ${ }^{14,50}$, Jenny A. Visser ${ }^{10}$, Ken K. Ong ${ }^{1,176}$, Jenny Chang-

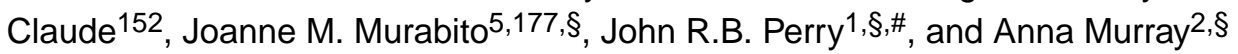

\section{Affiliations}

${ }^{1}$ MRC Epidemiology Unit, University of Cambridge School of Clinical Medicine, Box 285 Institute of Metabolic Science, Cambridge Biomedical Campus, Cambridge, CB2 OQQ, UK ${ }^{2}$ Genetics of Complex Traits, University of Exeter Medical School, University of Exeter, Exeter, EX2 5DW, UK ${ }^{3}$ Centre for Cancer Genetic Epidemiology, Department of Public Health and Primary Care, University of Cambridge, CB1 8RN, UK ${ }^{4}$ Boston University School of Public Health, Department of Biostatistics. Boston, Massachusetts 02118, USA ${ }^{5} \mathrm{NHLBl's} \mathrm{and} \mathrm{Boston}$ University's Framingham Heart Study, Framingham, Massachusetts 01702-5827, USA ${ }^{6}$ Estonian Genome Center, University of Tartu, Tartu, 51010, Estonia ${ }^{7}$ Institute of Molecular and Cell Biology, University of Tartu, Tartu 51010, Estonia ${ }^{8}$ Division of Preventive Medicine, Brigham and Women's Hospital, Boston, MA $02215{ }^{9}$ Harvard Medical School, Boston, MA 02115, USA ${ }^{10}$ Department of Internal Medicine, Erasmus MC, 3015GE Rotterdam, the Netherlands ${ }^{11}$ Netherlands Consortium on Health Aging and National Genomics Initiative, 2300 RC Leiden, the Netherlands ${ }^{12}$ Department of Epidemiology, Harvard School of Public Health, Boston, MA 02115, USA ${ }^{13}$ Department of Mathematics, Massachusetts Institute of Technology, Cambridge, Massachusetts 02139-4307, USA ${ }^{14}$ deCODE genetics/Amgen, Inc., IS-101 Reykjavik, Iceland ${ }^{15}$ Stanley Center for Psychiatric Research, Broad Institute of MIT and Harvard, Cambridge, Massachusetts 02142, USA ${ }^{16}$ Analytic and Translational Genetics Unit, Department of Medicine, Massachusetts General Hospital, Boston, MA, USA ${ }^{17}$ Medical and Population Genetics, Broad Institute, Cambridge, MA 02142, US ${ }^{18}$ Division of Endocrinology, Boston Children's Hospital, Boston, MA 02115, USA ${ }^{19}$ Department of Genetics, Harvard Medical School, Boston, MA 02115, USA ${ }^{20}$ Broad Institute of the Massachusetts Institute of Technology and Harvard University, 140 Cambridge 02142, MA, USA ${ }^{21}$ Department of Epidemiology, University of North Carolina, Chapel Hill, NC 27599, USA ${ }^{22}$ Department of Epidemiology, Indiana University Richard M. Fairbanks School of Public Health, Indianapolis, IN 46202, USA ${ }^{23}$ Indiana University Melvin and Bren Simon Cancer Center, Indianapolis, IN 46202, USA ${ }^{24}$ Research Unit of Molecular Epidemiology, Helmholtz Zentrum München - German Research Center for Environmental Health, 85764 Neuherberg, Germany ${ }^{25}$ Institute of Genetic Epidemiology, Helmholtz Zentrum München - German Research Center for Environmental Health, 85764 Neuherberg, Germany ${ }^{26}$ Institute of Epidemiology II, Helmholtz Zentrum München - German Research Center for Environmental Health, 85764 Neuherberg, Germany ${ }^{27}$ Cardiovascular Health Research Unit, Department of Medicine, University of Washington, Seattle Washington 98101 USA ${ }^{28}$ Department of Genetics, University of Groningen, University Medical Centre Groningen, Groningen, The Netherlands ${ }^{29}$ Medical Research Council Human 
Genetics Unit, Institute of Genetics and Molecular Medicine, University of Edinburgh, Edinburgh EH4 2XU, UK ${ }^{30}$ Merck Pharmaceuticals, 33 Avenue Louis Pasteur, Boston, MA 02115, United States ${ }^{31}$ Program in Personalized Medicine, Division of Endocrinology, Diabetes and Nutrition - University of Maryland School of Medicine, USA. Baltimore, MD $21201{ }^{32}$ Institute of Genetics and Biophysics - CNR, via Pietro Castellino 111, 80131, Naples, Italy ${ }^{33}$ Institute of Genetics and Biomedical Research, National Research Council, Cagliari, 09042 Sardinia, Italy ${ }^{34}$ University of Sassari, Department of Biomedical Sciences, Sassari, 07100 Sassari, Italy ${ }^{35}$ Center for Statistical Genetics, Ann Arbor, University of Michigan, Michigan 48109-2029, USA ${ }^{36}$ Institute for Maternal and Child Health - IRCCS "Burlo Garofolo", 34137 Trieste, Italy ${ }^{37}$ Fred Hutchinson Cancer Research Center, Public Health Sciences Division, Seattle, WA 98109-1024, USA ${ }^{38}$ Department of Epidemiology, School of Public Health, University of Michigan, Ann Arbor, MI, USA ${ }^{39}$ Institute for Community Medicine, University Medicine Greifswald, 17475 Greifswald, Germany ${ }^{40}$ Division of Genetics and Cell Biology, San Raffaele Scientific Institute, 20132 Milano, Italy ${ }^{41}$ Department of Clinical Medical Sciences, Surgical and Health, University of Trieste, 34149 Trieste, Italy ${ }^{42}$ The Institute for Translational Genomics and Population Sciences, Department of Pediatrics, LABioMed at Harbor-UCLA Medical Center, Torrance, California, USA ${ }^{43}$ Genetic Epidemiology Unit Department of Epidemiology, Erasmus MC, 3015 GE, Rotterdam, the Netherlands ${ }^{44}$ Department of Medical Genetics, University of Lausanne, $\mathrm{CH}-1005$ Lausanne, Switzerland ${ }^{45}$ Swiss Institute of Bioinformatics, $\mathrm{CH}-1015$, Lausanne, Switzerland ${ }^{46}$ Department of Biological Psychology, VU University Amsterdam, van der Boechorststraat 1, 1081 BT, Amsterdam, The Netherlands ${ }^{47}$ Department of Twin Research and Genetic Epidemiology, King's College London, London SE1 7EH, UK ${ }^{48}$ National Institute for Health Research (NIHR) Biomedical Research Centre at Guy's and St. Thomas' Foundation Trust, London, UK ${ }^{49}$ Icelandic Heart Association, Kopavogur, Iceland ${ }^{50}$ Faculty of Medicine, University of Iceland, IS-101 Reykjavik, Iceland ${ }^{51}$ Longitudinal Studies Section, Translational Gerontology Branch, National Institute on Aging, Baltimore, Maryland 21224, United States of America ${ }^{52}$ Lunenfeld-Tanenbaum Research Institute of Mount Sinai Hospital, Toronto, Ontario M5G 1X5, Canada ${ }^{53}$ Department of Molecular Genetics, University of Toronto, Toronto, Ontario, Canada ${ }^{54}$ Department of Epidemiology, University of California Irvine, Irvine, California, USA ${ }^{55}$ Division of Clinical Epidemiology and Aging Research, German Cancer Research Center (DKFZ), Heidelberg, Germany ${ }^{56}$ Department of Biostatistics, University of Washington, Seattle, WA, USA ${ }^{57}$ Department of Gynaecology and Obstetrics, University Hospital Erlangen, Friedrich-Alexander University Erlangen-Nuremberg, Erlangen, Germany ${ }^{58}$ Division of Epidemiology, Department of Medicine, Vanderbilt-Ingram Cancer Center, Vanderbilt University School of Medicine, Nashville, TN, USA ${ }^{59}$ Human Genetics Group, Human Cancer Genetics Program, Spanish National Cancer Research Centre (CNIO), Madrid, Spain ${ }^{60}$ Centro de Investigación en Red de Enfermedades Raras (CIBERER), Valencia, Spain ${ }^{61}$ Beckman Research Institute of City of Hope, Duarte, CA, USA ${ }^{62}$ Division of Epidemiology, Department of Health Sciences Research, Mayo Clinic, 
Rochester, Minnesota, USA ${ }^{63}$ Department of Oncology, University of Helsinki and Helsinki University Central Hospital, Helsinki, Finland ${ }^{64}$ Human Genetics Center, School of Public Health, The University of Texas Health Science Center at Houston, Houston, TX 77030, USA ${ }^{65}$ Human Genome Sequencing Center, Baylor College of Medicine, Houston, TX 77030, USA ${ }^{66}$ Department of Radiation Oncology, Hannover Medical School, Hannover, Germany ${ }^{67}$ Faculty of Health and Medical Sciences, University of Copenhagen, Copenhagen, Denmark ${ }^{68}$ Department of Clinical Biochemistry, Herlev Hospital, Copenhagen University Hospital, University of Copenhagen, Copenhagen, Denmark ${ }^{69}$ Department of Genetics, Institute for Cancer Research, Radiumhospitalet, Oslo University Hospital, Oslo University Hospital, Oslo, Norway ${ }^{70}$ Institute of Clinical Medicine, Faculty of Medicine, University of Oslo, Oslo, Norway ${ }^{71}$ Dr. Margarete Fischer-Bosch-Institute of Clinical Pharmacology, Stuttgart, Germany ${ }^{72}$ University of Tübingen, Tübingen, Germany ${ }^{73}$ German Cancer Consortium (DKTK), German Cancer Research Center (DKFZ), Heidelberg, Germany ${ }^{74}$ Division of Preventive Oncology, German Cancer Research Center (DKFZ), Heidelberg, Germany ${ }^{75}$ Institute for Prevention and Occupational Medicine of the German Social Accident Insurance, Institute of the Ruhr University Bochum (IPA), Bochum, Germany ${ }^{76}$ Division of Molecular Genetic Epidemiology, German Cancer Research Center, Heidelberg, Germany ${ }^{77}$ Molecular Biology of Breast Cancer, Department of Obstetrics and Gynecology, University of Heidelberg, Heidelberg, Germany ${ }^{78}$ Medical Genetics Section, Centre for Genomic and Experimental Medicine, Institute of Genetics and Molecular Medicine, University of Edinburgh, Edinburgh EH4 2XU, UK ${ }^{79}$ Institute for Population Health Sciences and Informatics, University of Edinburgh, Teviot Place, Edinburgh, EH8 9AG, Scotland ${ }^{80}$ Division of Cancer Epidemiology and Genetics, National Cancer Institute, Bethesda, MD, USA ${ }^{81}$ Wellcome Trust Centre for Human Genetics, University of Oxford, Oxford, UK ${ }^{82}$ Department of Genetics, QIMR Berghofer Medical Research Institute, Brisbane, Australia ${ }^{83}$ Department of Laboratory Medicine and Pathology, Mayo Clinic, Rochester, MN, USA ${ }^{84}$ Boston University School of Medicine, Department of Medicine, Sections of Preventive Medicine and Endocrinology, Boston, MA ${ }^{85}$ Sheffield Cancer Research, Department of Oncology, University of Sheffield, Sheffield, UK ${ }^{86}$ Department of Medical Epidemiology and Biostatistics, Karolinska Institutet, 17177 Stockholm, Sweden ${ }^{87}$ Channing Division of Network Medicine, Department of Medicine, Brigham and Women's Hospital and Harvard Medical School, Boston, MA 02115, USA ${ }^{88}$ Division of Epidemiology \& Community Health, University of Minnesotta, Minneapolis MN $55455{ }^{89}$ Department of Human Genetics, Leiden University Medical Center, 2300 RC Leiden, The Netherlands ${ }^{90}$ Department of Pathology, Leiden University Medical Center, 2300 RC Leiden, The Netherlands ${ }^{91}$ Gynaecology Research Unit, Hannover Medical School, Hannover, Germany ${ }^{92}$ Non-communicable Disease Epidemiology Department, London School of Hygiene and Tropical Medicine, London, UK ${ }^{93}$ Centre for Cancer Genetic Epidemiology, Department of Oncology, University of Cambridge, Cambridge, CB1 8RN, UK ${ }^{94}$ David Geffen School of Medicine, Department of Medicine Division of Hematology and Oncology, University of California at Los Angeles, CA, USA 
${ }^{95}$ Survey Research Center, Institute for Social Research, University of Michigan, Ann Arbor, MI, USA ${ }^{96}$ Division of Cancer Epidemiology and Genetics, National Cancer Institute, Rockville, MD, USA ${ }^{97}$ Department of Cancer Epidemiology/Clinical Cancer Registry, University Clinic Hamburg-Eppendorf, Hamburg, Germany ${ }^{98}$ Institute for Medical Biometrics and Epidemiology, University Clinic HamburgEppendorf, Hamburg, Germany ${ }^{99}$ Laboratory of Epidemiology and Population Sciences, National Institute on Aging, Bethesda, MD, USA ${ }^{100}$ Division of Genetics and Epidemiology, The Institute of Cancer Research, London, UK ${ }^{101}$ Division of Cancer Studies, Breakthrough Breast Cancer Research Centre, The Institute of Cancer Research, London, UK ${ }^{102}$ Centre for Epidemiology and Biostatistics, Melbourne School of Population and Global Health, The University of Melbourne, Melbourne, Australia ${ }^{103}$ Cancer Epidemiology Centre, Cancer Council Victoria, Melbourne, Australia ${ }^{104}$ Department of Medicine, McGill University, Montreal, Canada ${ }^{105}$ Division of Clinical Epidemiology, Royal Victoria Hospital, McGill University, Montreal, Canada ${ }^{106}$ Division of Endocrinology, Diabetes and Metabolism, Cedars-Sinai Medical Center, Los Angeles, California, USA ${ }^{107}$ School of Engineering and Natural Sciences, University of Iceland, IS-101 Reykjavik, Iceland, ${ }^{108}$ Environmental Epidemiology of Cancer, Center for Research in Epidemiology and Population Health, INSERM, Villejuif, France ${ }^{109}$ University ParisSud, UMRS 1018, Villejuif, France ${ }^{110}$ Department of Preventive Medicine, Keck School of Medicine, University of Southern California, Los Angeles, CA, USA ${ }^{111}$ Molecular Genetics of Breast Cancer, Deutsches Krebsforschungszentrum (DKFZ), Heidelberg, Germany ${ }^{112}$ Musculoskeletal Research Programme, Division of Applied Medicine, University of Aberdeen, Aberdeen AB25 2ZD, UK ${ }^{113}$ Interfaculty Institute for Genetics and Functional Genomics, University Medicine Greifswald, 17475 Greifswald, Germany ${ }^{114}$ Department of Medical Oncology, Erasmus University Medical Center, Rotterdam, The Netherlands ${ }^{115}$ Department of Nutrition, Harvard School of Public Health, Boston, MA 02115, USA ${ }^{116}$ State Key Laboratory of Medical Genomics, Shanghai Institute of Hematology, Rui Jin Hospital, Shanghai Jiao Tong University School of Medicine, Shanghai 200025, China ${ }^{117}$ Department of Genetics and Pathology, Pomeranian Medical University, Szczecin, Poland ${ }^{118}$ Hebrew SeniorLife Institute for Aging Research, Boston, MA, 02131, USA ${ }^{119}$ Prosserman Centre for Health Research, Lunenfeld-Tanenbaum Research Institute of Mount Sinai Hospital, Toronto, Canada ${ }^{120}$ Division of Epidemiology, Dalla Lana School of Public Health, University of Toronto, Toronto, Ontario, Canada ${ }^{121}$ Faculty of Medicine, University of Split, Split, Croatia ${ }^{122}$ Cancer Center, Kuopio University Hospital, Kuopio, Finland ${ }^{123}$ School of Medicine, Institute of Clinical Medicine, Pathology and Forensic Medicine, University of Eastern Finland, Kuopio, Finland ${ }^{124}$ Imaging Center, Department of Clinical Pathology, Kuopio University Hospital, Kuopio, Finland ${ }^{125}$ German Center for Diabetes Research, 85764 Neuherberg, Germany ${ }^{126}$ Department of Clinical Molecular Biology, Oslo University Hospital, University of Oslo, Oslo, Norway ${ }^{127}$ Vesalius Research Center (VRC), VIB, Leuven, Belgium ${ }^{128}$ Laboratory for Translational Genetics, Department of Oncology, University of Leuven, Leuven, Belgium ${ }^{129}$ Center for Human Genetics, Division of 
Public Health Sciences, Wake Forest School of Medicine ${ }^{130}$ Department of Oncology - Pathology, Karolinska Institutet, Stockholm 17177, Sweden ${ }^{131}$ QIMR Berghofer Medical Research Institute, Brisbane, Queensland, Australia ${ }^{132}$ Division of Gynaecology and Obstetrics, Technische Universität München, Munich, Germany ${ }^{133}$ Department of Medicine I, Ludwig-Maximilians-University Munich, 81377 Munich, Germany ${ }^{134}$ DZHK (German Centre for Cardiovascular Research), partner site Munich Heart Alliance, Munich, Germany ${ }^{135}$ Laboratory of Neurogenetics, National Institute on Aging, Bethesda, MD, USA ${ }^{136}$ Department of Obstetrics and Gynecology, University of Helsinki and Helsinki University Central Hospital, Helsinki, Finland ${ }^{137}$ KULeuven (University of Leuven), Department of Oncology, Multidisciplinary Breast Center, University Hospitals Leuven, Belgium ${ }^{138}$ Department of Epidemiology, University of Pittsburgh, Pittsburgh, Pennsylvania, USA ${ }^{139}$ Department of Medicine, University of Pittsburgh, Pittsburgh, Pennsylvania, USA ${ }^{140}$ Department of Clinical and Translational Science, University of Pittsburgh, Pittsburgh, Pennsylvania, USA ${ }^{141}$ British Heart Foundation Glasgow Cardiovascular Research Centre, Institute of Cardiovascular and Medical Sciences, College of Medical, Veterinary and Life Sciences, University of Glasgow, Glasgow G12 8TA, UK ${ }^{142}$ IFOM, Fondazione Istituto FIRC di Oncologia Molecolare, Milan, Italy ${ }^{143}$ Institute of Clinical Chemistry and Laboratory Medicine, University Medicine Greifswald, 17475 Greifswald, Germany ${ }^{144}$ Department of Epidemiology, School of Public Health, University of Washington, Seattle, WA 98195, USA ${ }^{145}$ Group Health Research Institute, Group Health Cooperative, Seattle, Washington 98101, USA ${ }^{146}$ Department of Health Services, University of Washington, Seattle, Washington 98101, USA ${ }^{147}$ Laboratory of Cancer Genetics and Tumor Biology, Department of Clinical Chemistry, University of Oulu, Oulu, Finland ${ }^{148}$ Laboratory of Cancer Genetics and Tumor Biology, Northern Finland Laboratory Centre NordLab, Oulu, Finland ${ }^{149}$ Unit of Molecular Bases of Genetic Risk and Genetic Testing, Department of Preventive and Predictive Medicine, Fondazione IRCCS Istituto Nazionale dei Tumori (INT), Milan, Italy ${ }^{150}$ Medical Genetics Research Institute, Cedars-Sinai Medical Center, Los Angeles, California, USA ${ }^{151}$ UCLA Clinical \& Translational Science Institute, Cedars-Sinai Medical Center, Los Angeles, California, USA ${ }^{152}$ Division of Cancer Epidemiology, German Cancer Research Center (DKFZ), Heidelberg, Germany ${ }^{153}$ Research Oncology, Guy's Hospital, King's College London, London, UK ${ }^{154}$ National Institute on Aging, Intramural Research Program, Baltimore, MD 20892, USA ${ }^{155}$ Netherlands Cancer Institute, Antoni van Leeuwenhoek hospital, Amsterdam, The Netherlands ${ }^{156}$ Division of Molecular Gyneco-Oncology, Department of Gynaecology and Obstetrics, University Hospital of Cologne, Cologne, Germany ${ }^{157}$ Center of Familial Breast and Ovarian Cancer, University Hospital of Cologne, Cologne, Germany ${ }^{158}$ Center for Integrated Oncology, University Hospital of Cologne, Cologne, Germany ${ }^{159}$ Centre Hospitalier Universitaire de Québec Research Center, Laval University, Québec City, Canada ${ }^{160}$ Department of Pathology, The University of Melbourne, Melbourne, Australia ${ }^{161}$ Institute of Medical Informatics, Biometry and Epidemiology, Chair of Genetic Epidemiology, Ludwig-Maximilians-Universität, 81377 Munich, Germany ${ }^{162}$ Division 
of Breast Cancer Research, The Institute of Cancer Research, London, UK ${ }^{163}$ Department of Molecular Virology, Immunology and Medical Genetics, Comprehensive Cancer Center, The Ohio State University, Columbus, OH, USA ${ }^{164}$ NIHR Oxford Biomedical Research Centre, Churchill Hospital, OX3 7LE Oxford, UK ${ }^{165}$ Icelandic Cancer Registry, Reykjavik, Iceland ${ }^{166}$ Division of Nephrology and Hypertension, Department of Internal Medicine, Mayo Clinic, Rochester, MN, USA ${ }^{167}$ Department of Medicine, Vanderbilt University Medical Center, Nashville, Tennessee, 37203, USA ${ }^{168}$ Department of Endocrinology, University of Groningen, University Medical Centre Groningen, Groningen, The Netherlands ${ }^{169}$ LifeLines Cohort Study and Biobank, University Medical Center Groningen, University of Groningen, Groningen, The Netherlands ${ }^{170}$ Molecular Diagnostics Laboratory, IRRP, National Centre for Scientific Research "Demokritos", Athens, Greece ${ }^{171}$ Department of Obstetrics and Gynecology, University Medicine Greifswald, 17475 Greifswald, Germany ${ }^{172}$ Department of Epidemiology, University of Groningen, University Medical Center Groningen, Groningen, The Netherlands ${ }^{173}$ Department of Biostatistics, Harvard School of Public Health, Boston, MA 02115, USA ${ }^{174}$ Peter MacCallum Cancer Centre, The University of Melbourne, Melbourne, Australia ${ }^{175} \mathrm{~A}$ Collaboration between the University Medical Schools and NHS in Aberdeen, Dundee, Edinburgh and Glasgow, UK ${ }^{176}$ Department of Paediatrics, University of Cambridge,Cambridge, CB2 0QQ, UK ${ }^{177}$ Boston University School of Medicine, Department of Medicine, Section of General Internal Medicine, Boston, MA 02118, USA

\section{Acknowledgements}

See supplementary information

\section{URLS}

http://www.ons.gov.uk/ons/publications/

www.hgmd.cf.ac.uk/

http://research.nhgri.nih.gov/bic/

http://www.genome.gov/gwastudies/)

http://www.chargeconsortium.com/main/exomechip

http://string-db.org/

http://www.broadinstitute.org/mpg/snap/

https://github.com/bulik/ldsc

http://www.1000genomes.org/ 
https://github.com/bulik/ldsc

\section{Author contributions}

All authors reviewed the original and revised manuscripts.

Statistical Analysis

F.R.D, K.S.R, D.J.T, K.L.L, N.P, D.I.C, L.S, H.K.F, P.S, B.B-S, T.E, A.D.J, C.E.E, N.F, C.He, E.Alt, J.A.B, L.L.F, J.E.H, S.E.J, M.F.K, P.F.M, T.N, E.P, A.Ro, L.M.R, U.M.S, J.A.S, A.T, M.T, D.Vu, J.Y, W.Zhao, E.Alb, N.A, T.C, J-J.H, M.Ma, A.V.S, T.Ta, J.R.B.P

\section{Sample collection, genotyping and phenotyping}

G.A, I.L.A, H.A, A.C.A, V.A, A.M.A, C.Ba, M.W.B, A.B-F, J.B, L.B, S.J.B, C.Bl, E.B, N.V.B, S.E.B, M.K.B, A.B-D, T.S.B, H.Bra, H.Bre, T.B, B.B, A.Ca, H.C, S.J.C, J.R.C, Y.C, G.C, F.J.C, A.D.C, A.Co, K.C, H.D, I.DV, E.W.D, J.D, P.D, T.D, I.dSS, A.M.D, J.D.E, P.A.F, J.D.F, J.F, D.F, I.G, M.E.G, M.G, G.G.Giles, G.G.G, M.S.G, A.G, M.O.G, M.L.G, D.F.G, P.G, X.G, C.A.H, P.H, U.H, B.E.H, L.J.H, A.H, G.H, M.J.H, J.L.H, F.B.H, J.H, K.H, D.J.H, A.J, M.K, D.K, J.A.K, I.K, C.K, V.Ko, J.K, V.Kr, D.L, C.L, J.Li, X.L, S.L, Y.L, J.Lua, J.Lub, R.M, A.Ma, J.Manz, S.M, J.M, N.G. M, C.M, A.Mei, K.M, E.M, L.M, R.L.M, M.Mü, M.N, B.M.N, H.N, P.N, A.B.N, B.G.N, J.E.O, S.P, P.P, U.P, A.Pe, J.P, P.D.P.P, N.N.P, A.Pi, G.P, O.P, D.P, B.M.P, K.P, P.R, L.J. R, F.R, I.R, A.Ru, D.R, C.F.S, S.S, E.J.S, D.Sc, M.K.S, F.S, R.K.S, M.J.S, R.A.S, C.MS, J.S, R.S, M.C.S, D.St, K.Str, A.S, K.D.T, U.T, A.E.T, I.T, T.Tr, L.T, S.T.T, D.Vo, Q.W, M.W, G.W, J.F.W, R.W, B.B.H.R.W, A.F.W, D.Y, T.Z, W.Z, M.Z

\section{Individual study PI}

S.B, D.I.B, J.E.B, L.F, G.W.M, V.G, T.D.S, C.Mv, B.Z.A, M.C, L.C, D.F.E, P.P.G, C.G, T.B.H, C.Ha, S.L.R.K, P.K, B.M, A.Met, A.C.M, A.P.R, P.M.R, J.I.R, D.T, A.G.U, S.U, H.V, N.J.W, D.R.W, L.M.Y, A.L.P, K.Ste, J.A.V, K.K.O, J.C-C, J.M.M, A.Mu

\section{Working group}

F.R.D, K.S.R, D.J.T, K.L.L, N.P, D.I.C, L.S, H.K.F, P.S, B.B-S, T.E, A.D.J, C.E.E, N.F, C.He, A.L.P, K.Ste, J.A.V, K.K.O, J.C-C, J.M.M, J.R.B.P, A.Mu

\section{References}

1. Hartge P. Genetics of reproductive lifespan. Nat Genet. 2009; 41:637-638. [PubMed: 19471299]

2. Lambalk CB, van Disseldorp J, de Koning CH, Broekmans FJ. Testing ovarian reserve to predict age at menopause. Maturitas. 2009; 63:280-91. [PubMed: 19631481]

3. te Velde ER, Pearson PL. The variability of female reproductive ageing. Hum Reprod Update. 2002; 8:141-54. [PubMed: 12099629]

4. Stolk L, et al. Meta-analyses identify 13 loci associated with age at menopause and highlight DNA repair and immune pathways. Nature genetics. 2012; 44:260-8. [PubMed: 22267201]

5. Perry JR, et al. DNA mismatch repair gene MSH6 implicated in determining age at natural menopause. Hum Mol Genet. 2014 [PubMed: 24357391] 
6. Finucane HK, et al. Partitioning heritability by functional category using GWAS summary statistics. 2015

7. Oktem O, Oktay K. The ovary: anatomy and function throughout human life. Ann N Y Acad Sci. 2008; 1127:1-9. [PubMed: 18443323]

8. Guler GD, et al. Human DNA helicase B (HDHB) binds to replication protein A and facilitates cellular recovery from replication stress. J Biol Chem. 2012; 287:6469-81. [PubMed: 22194613]

9. Weischer M, Bojesen SE, Ellervik C, Tybjaerg-Hansen A, Nordestgaard BG. CHEK2*1100delC genotyping for clinical assessment of breast cancer risk: meta-analyses of 26,000 patient cases and 27,000 controls. J Clin Oncol. 2008; 26:542-8. [PubMed: 18172190]

10. AlAsiri S, et al. Exome sequencing reveals MCM8 mutation underlies ovarian failure and chromosomal instability. J Clin Invest. 2014

11. Fogli A, et al. Ovarian failure related to eukaryotic initiation factor $2 \mathrm{~B}$ mutations. Am J Hum Genet. 2003; 72:1544-50. [PubMed: 12707859]

12. Trifunovic A, et al. Premature ageing in mice expressing defective mitochondrial DNA polymerase. Nature. 2004; 429:417-23. [PubMed: 15164064]

13. Mandon-Pepin B, et al. Genetic investigation of four meiotic genes in women with premature ovarian failure. Eur J Endocrinol. 2008; 158:107-15. [PubMed: 18166824]

14. Linder B, et al. Tdrd 3 is a novel stress granule-associated protein interacting with the Fragile-X syndrome protein FMRP. Hum Mol Genet. 2008; 17:3236-46. [PubMed: 18664458]

15. Eicher JD, et al. GRASP v2.0: an update on the Genome-Wide Repository of Associations between SNPs and phenotypes. Nucleic Acids Res. 2014

16. Morris DH, et al. Body mass index, exercise, and other lifestyle factors in relation to age at natural menopause: analyses from the breakthrough generations study. Am J Epidemiol. 175:998-1005. [PubMed: 22494951]

17. Perry JR, et al. Parent-of-origin-specific allelic associations among 106 genomic loci for age at menarche. Nature. 2014; 514:92-7. [PubMed: 25231870]

18. Collaborative Group on Hormonal Factors in Breast, C. Menarche, menopause, and breast cancer risk: individual participant meta-analysis, including 118964 women with breast cancer from 117 epidemiological studies. Lancet Oncol. 2012; 13:1141-51. [PubMed: 23084519]

19. Vimaleswaran KS, et al. Association of vitamin D status with arterial blood pressure and hypertension risk: a mendelian randomisation study. Lancet Diabetes Endocrinol. 2014; 2:719-29. [PubMed: 24974252]

20. Michailidou K, et al. Large-scale genotyping identifies 41 new loci associated with breast cancer risk. Nat Genet. 2013; 45:353-61. 361e1-2. [PubMed: 23535729]

21. Dickson SP, Wang K, Krantz I, Hakonarson H, Goldstein DB. Rare variants create synthetic genome-wide associations. PLoS Biol. 2010; 8:e1000294. [PubMed: 20126254]

22. Monteiro AN, Freedman ML. Lessons from postgenome-wide association studies: functional analysis of cancer predisposition loci. J Intern Med. 2013; 274:414-24. [PubMed: 24127939]

23. Ghoussaini M, Pharoah PD, Easton DF. Inherited genetic susceptibility to breast cancer: the beginning of the end or the end of the beginning? Am J Pathol. 2013; 183:1038-51. [PubMed: 23973388]

24. Titus S, et al. Impairment of BRCA1-related DNA double-strand break repair leads to ovarian aging in mice and humans. Sci Transl Med. 2013; 5:172ra21.

25. Fan S, et al. BRCA1 inhibition of estrogen receptor signaling in transfected cells. Science. 1999; 284:1354-6. [PubMed: 10334989]

26. $\mathrm{Hu}$ Y, et al. RAP80-directed tuning of BRCA1 homologous recombination function at ionizing radiation-induced nuclear foci. Genes Dev. 2011; 25:685-700. [PubMed: 21406551]

27. Tsutsui Y, et al. Multiple regulation of Rad51-mediated homologous recombination by fission yeast Fbh1. PLoS Genet. 2014; 10:e1004542. [PubMed: 25165823]

28. Simandlova J, et al. FBH1 helicase disrupts RAD51 filaments in vitro and modulates homologous recombination in mammalian cells. J Biol Chem. 2013; 288:34168-80. [PubMed: 24108124]

29. Chapman JR, Taylor MR, Boulton SJ. Playing the end game: DNA double-strand break repair pathway choice. Mol Cell. 2012; 47:497-510. [PubMed: 22920291] 
30. Wood-Trageser MA, et al. MCM9 Mutations Are Associated with Ovarian Failure, Short Stature, and Chromosomal Instability. Am J Hum Genet. 2014; 95:754-62. [PubMed: 25480036]

31. Oktay K, Kim JY, Barad D, Babayev SN. Association of BRCA1 mutations with occult primary ovarian insufficiency: a possible explanation for the link between infertility and breast/ovarian cancer risks. J Clin Oncol. 2010; 28:240-4. [PubMed: 19996028]

32. Collins IM, et al. Do BRCA1 and BRCA2 mutation carriers have earlier natural menopause than their noncarrier relatives? Results from the Kathleen Cuningham Foundation Consortium for Research into Familial Breast Cancer. J Clin Oncol. 2013; 31:3920-5. [PubMed: 24081944]

33. Manson JE, et al. Menopausal hormone therapy and health outcomes during the intervention and extended poststopping phases of the Women's Health Initiative randomized trials. JAMA. 2013; 310:1353-68. [PubMed: 24084921]

34. Levy-Lahad E, Friedman E. Cancer risks among BRCA1 and BRCA2 mutation carriers. Br J Cancer. 2007; 96:11-5. [PubMed: 17213823]

35. Rance NE. Menopause and the human hypothalamus: evidence for the role of kisspeptin/ neurokinin B neurons in the regulation of estrogen negative feedback. Peptides. 2009; 30:111-22. [PubMed: 18614256]

36. Silveira LF, Latronico AC. Approach to the patient with hypogonadotropic hypogonadism. J Clin Endocrinol Metab. 2013; 98:1781-8. [PubMed: 23650335]

37. Garcia-Ortega J, et al. Expression of neurokinin B/NK3 receptor and kisspeptin/KISS1 receptor in human granulosa cells. Hum Reprod. 2014; 29:2736-46. [PubMed: 25316443]

38. Gaytan F, et al. Kisspeptin receptor haplo-insufficiency causes premature ovarian failure despite preserved gonadotropin secretion. Endocrinology. 2014; 155:3088-97. [PubMed: 24885574]

\section{Methods-only references}

39. Willer CJ, Li Y, Abecasis GR. METAL: fast and efficient meta-analysis of genomewide association scans. Bioinformatics. 2010; 26:2190-1. [PubMed: 20616382]

40. Purcell S, et al. PLINK: a tool set for whole-genome association and population-based linkage analyses. Am J Hum Genet. 2007; 81:559-75. [PubMed: 17701901]

41. Zhou JJ, et al. A comparative analysis of family-based and population-based association tests using whole genome sequence data. BMC Proc. 2014; 8:S33. [PubMed: 25519381]

42. Yang J, Lee SH, Goddard ME, Visscher PM. GCTA: a tool for genome-wide complex trait analysis. Am J Hum Genet. 2011; 88:76-82. [PubMed: 21167468]

43. Barrett JC, Fry B, Maller J, Daly MJ. Haploview: analysis and visualization of LD and haplotype maps. Bioinformatics. 2005; 21:263-5. [PubMed: 15297300]

44. Raychaudhuri $\mathrm{S}$, et al. Identifying relationships among genomic disease regions: predicting genes at pathogenic SNP associations and rare deletions. PLoS Genet. 2009; 5:e1000534. [PubMed: 19557189]

45. Zhang X, et al. Synthesis of 53 tissue and cell line expression QTL datasets reveals master eQTLs. BMC Genomics. 2014; 15:532. [PubMed: 24973796]

46. Segre AV, et al. Common inherited variation in mitochondrial genes is not enriched for associations with type 2 diabetes or related glycemic traits. PLoS Genet. 2010; 6 [PubMed: 20714348]

47. Hoffman MM, et al. Integrative annotation of chromatin elements from ENCODE data. Nucleic Acids Res. 2013; 41:827-41. [PubMed: 23221638]

48. Lindblad-Toh K, et al. A high-resolution map of human evolutionary constraint using 29 mammals. Nature. 2011; 478:476-82. [PubMed: 21993624]

49. Hnisz D, et al. Super-enhancers in the control of cell identity and disease. Cell. 2013; 155:934-47. [PubMed: 24119843]

50. Andersson R, et al. An atlas of active enhancers across human cell types and tissues. Nature. 2014; 507:455-61. [PubMed: 24670763]

51. Trynka G, et al. Chromatin marks identify critical cell types for fine mapping complex trait variants. Nat Genet. 2013; 45:124-30. [PubMed: 23263488] 
52. Schizophrenia Working Group of the Psychiatric Genomics, C. Biological insights from 108 schizophrenia-associated genetic loci. Nature. 2014; 511:421-7. [PubMed: 25056061]

53. Howie B, Fuchsberger C, Stephens M, Marchini J, Abecasis GR. Fast and accurate genotype imputation in genome-wide association studies through pre-phasing. Nat Genet. 2012; 44:955-9. [PubMed: 22820512]

54. Genomes Project, C. et al. An integrated map of genetic variation from 1,092 human genomes. Nature. 2012; 491:56-65. [PubMed: 23128226]

55. International Consortium for Blood Pressure Genome-Wide Association, S. et al. Genetic variants in novel pathways influence blood pressure and cardiovascular disease risk. Nature. 2011; 478:103-9. [PubMed: 21909115]

56. Eeles RA, et al. Identification of 23 new prostate cancer susceptibility loci using the iCOGS custom genotyping array. Nat Genet. 2013; 45:385-91. 391e1-2. [PubMed: 23535732]

57. Cross-Disorder Group of the Psychiatric Genomics, C. Identification of risk loci with shared effects on five major psychiatric disorders: a genome-wide analysis. Lancet. 2013; 381:1371-9. [PubMed: 23453885]

58. Bulik-Sullivan B, et al. An Atlas of Genetic Correlations across Human Diseases and Traits. 2015 [PubMed: 26414676]

59. Speliotes EK, et al. Association analyses of 249,796 individuals reveal 18 new loci associated with body mass index. Nat Genet. 2010; 42:937-48. [PubMed: 20935630] 


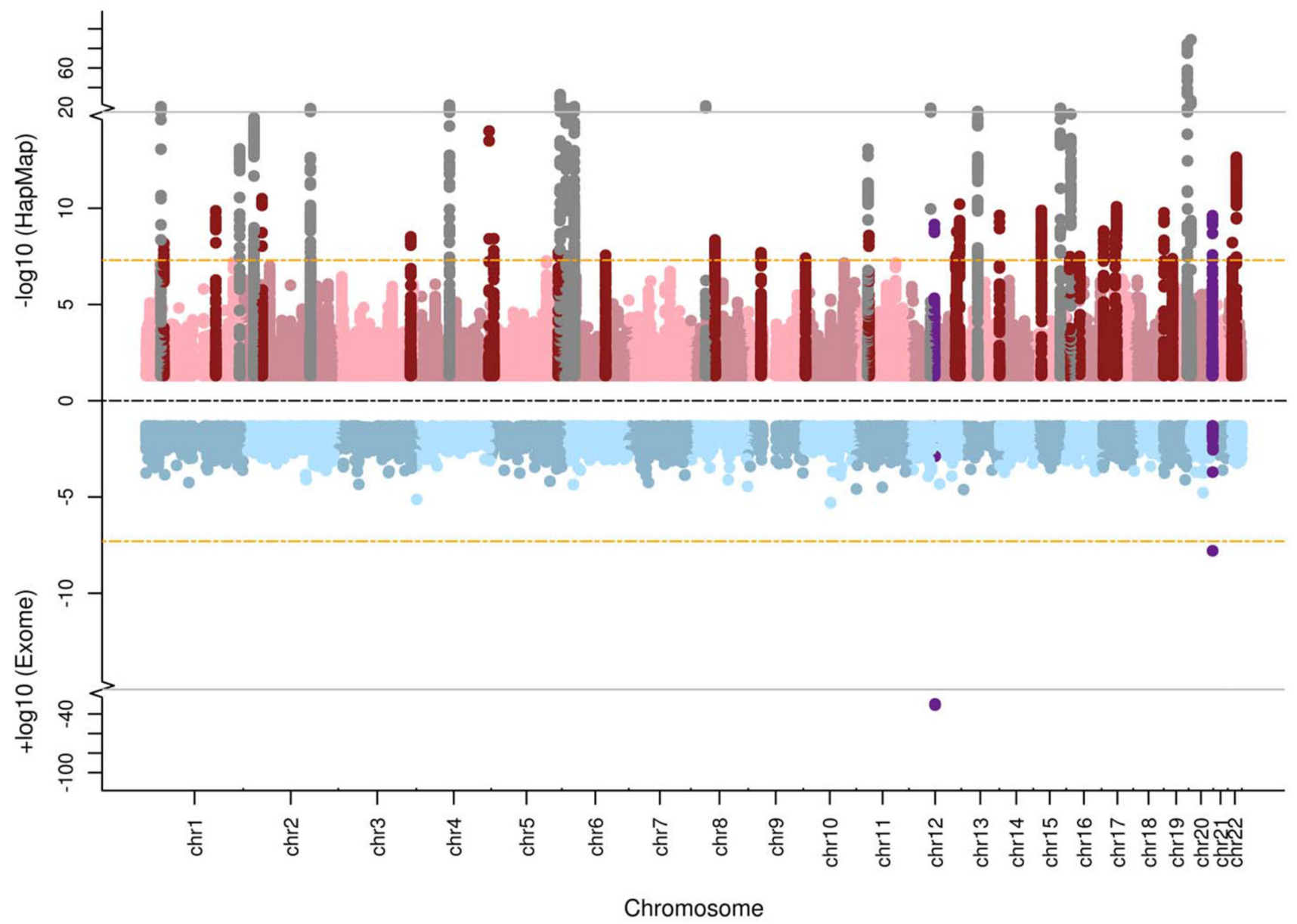

Figure 1.

Miami plot of HapMap and exome SNP associations. Log-transformed P values are shown for association with ANM for SNPs from HapMap 2 (top; pink) and SNPs from the metaanalysis of exome chip data (bottom; blue). Previously known signals are shown in gray, and newly discovered signals are shown in red (HapMap 2) or purple (exome chip and HapMap 2 ). The yellow lines correspond to genome-wide significant levels in each direction; the gray lines indicate where the y axis has been truncated. 


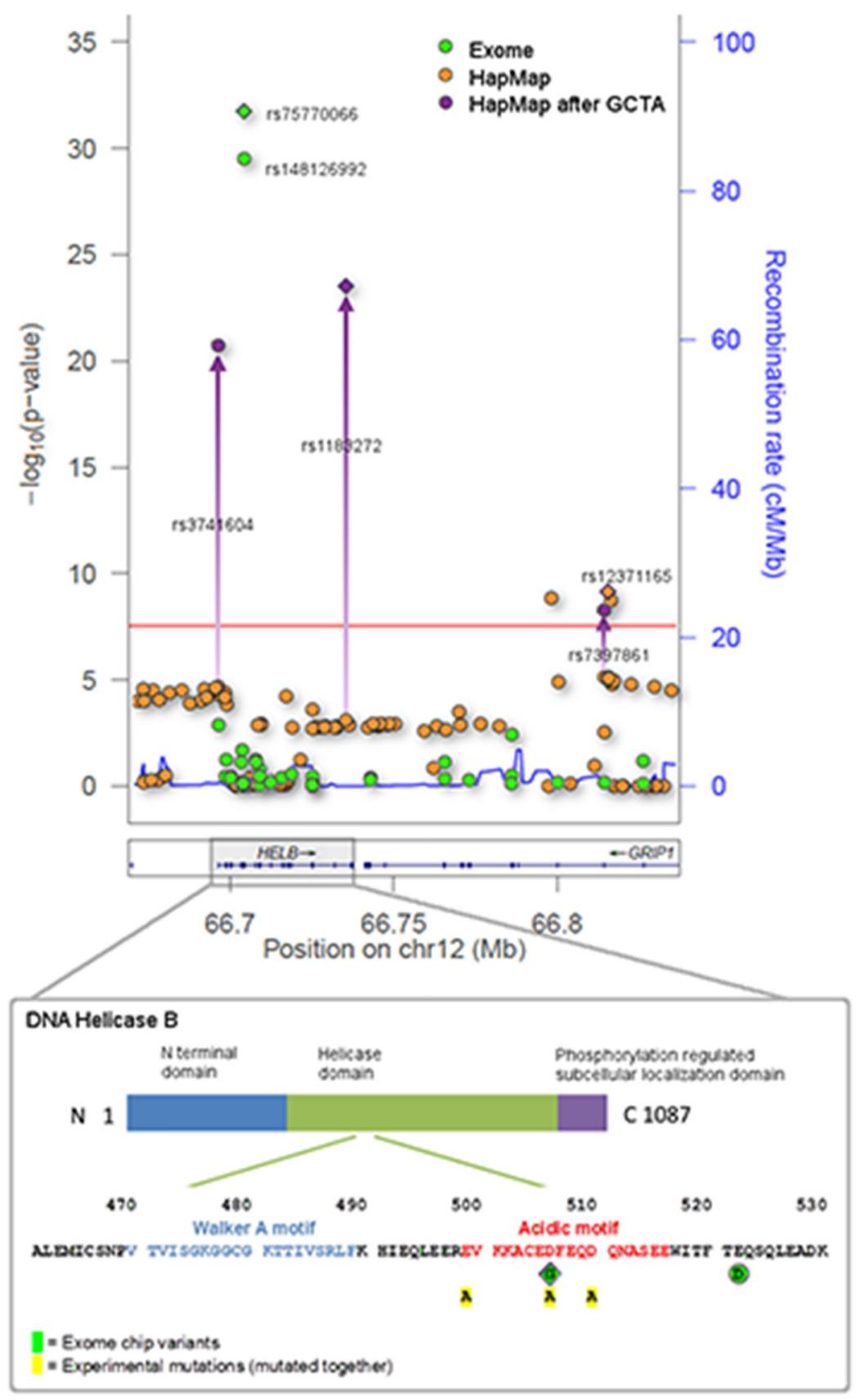

Figure 2.

Multiple signals at HELB and relationship to DNA helicase B protein sequence. Positions are given in Build 37 coordinates of the reference genome. The top signal from the exome chip analysis maps to an acidic motif of DNA helicase B and results in the replacement of an acidic aspartate residue by a nonpolar glycine residue. Concurrent alteration of three acidic amino acids, (including the aspartate residue identified by the exome chip analysis) to nonpolar residues has been shown to reduce RPA binding (8) 


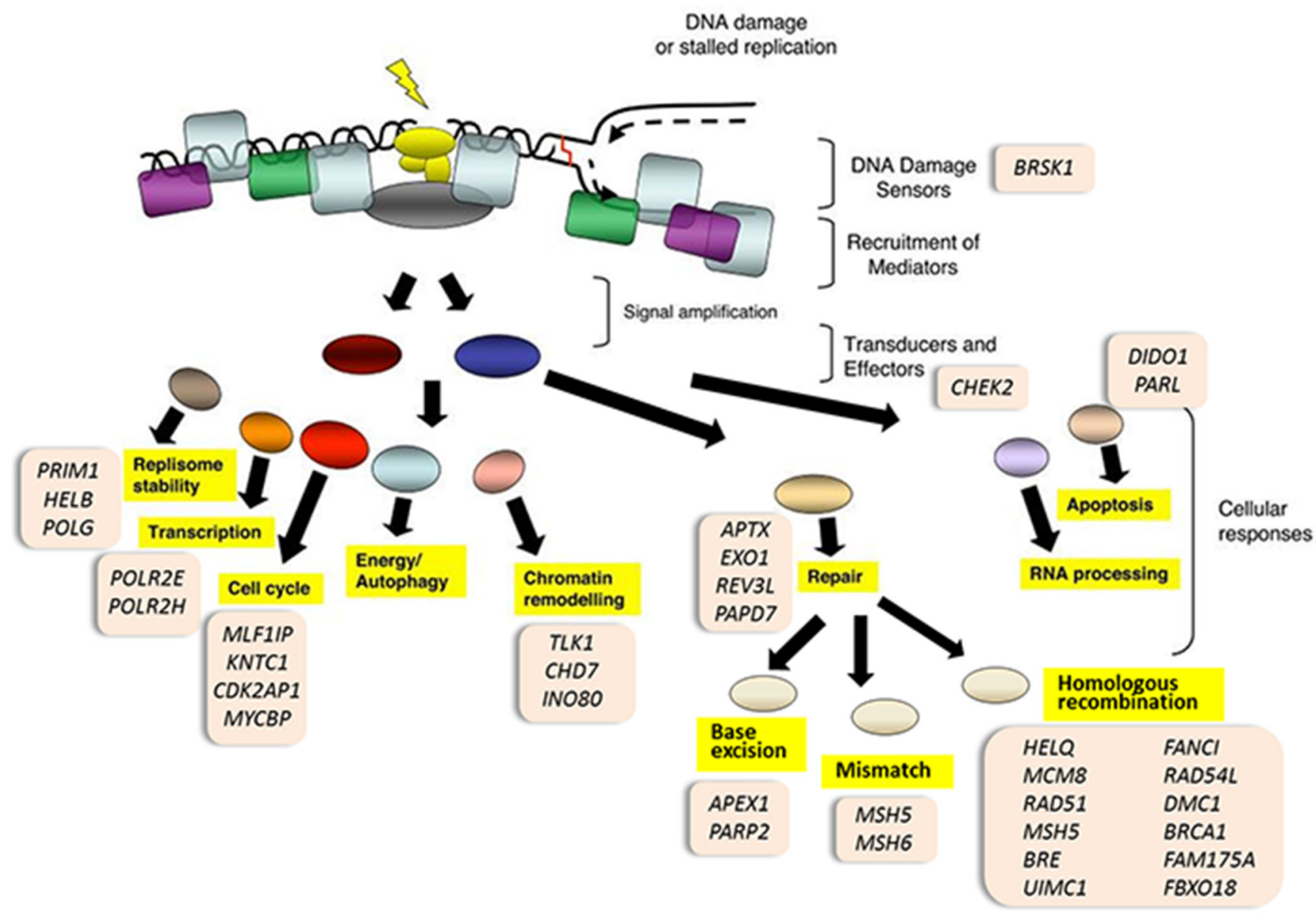

Adapted from: Jackson \& Bartek, Nature, 2009

Figure 3.

Classification of genes identified as being involved in the DNA damage response, at genetic loci associated with ANM. 


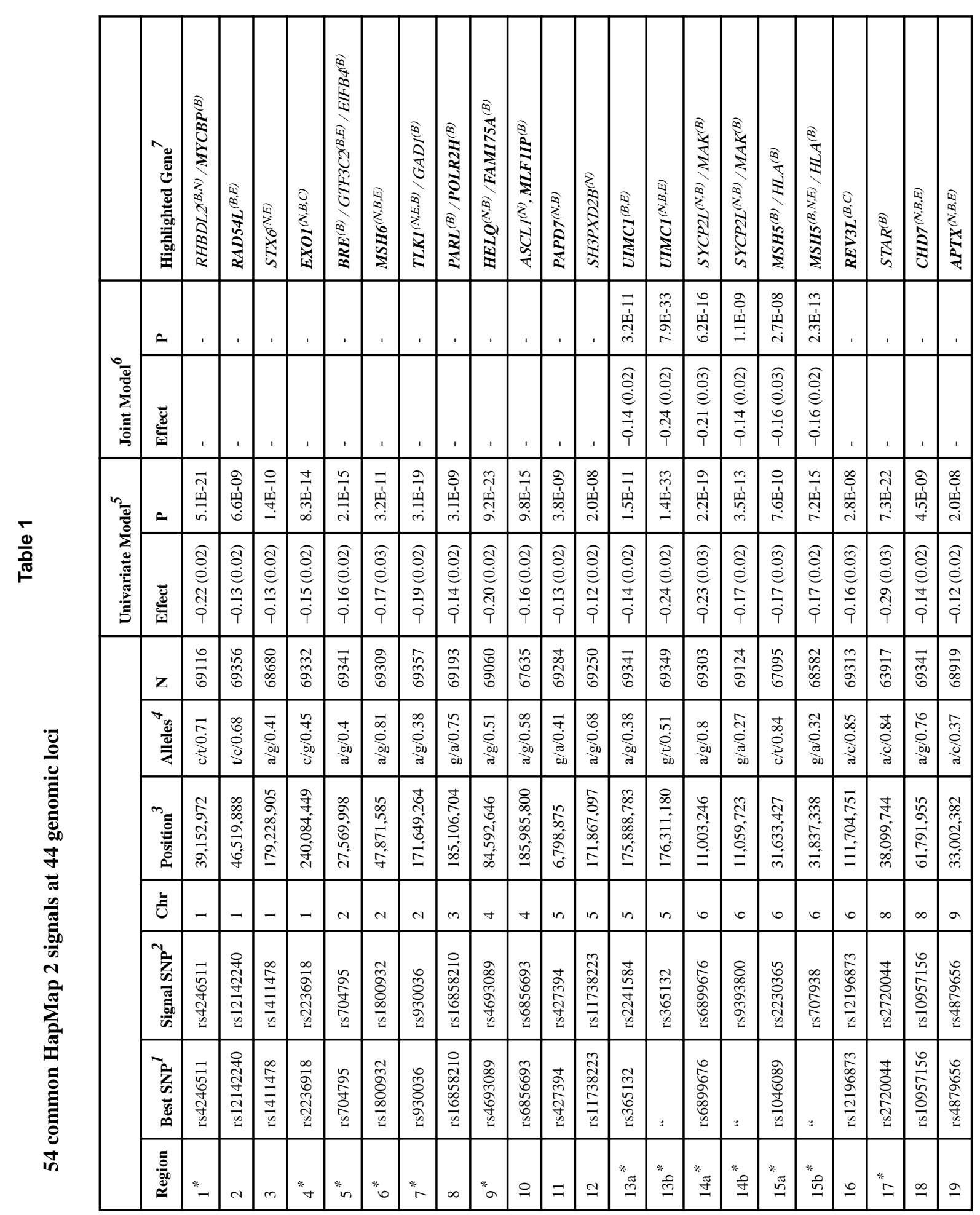




\begin{tabular}{|c|c|c|c|c|c|c|c|c|c|c|c|c|c|c|c|c|c|c|c|c|c|c|c|c|c|c|}
\hline & 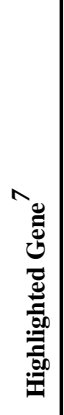 & 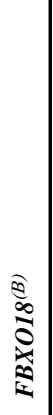 & 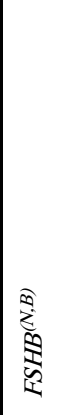 & 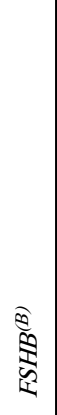 & 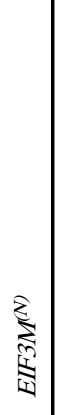 & 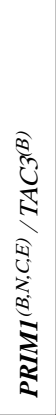 & 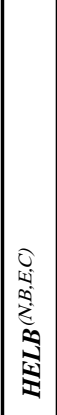 & 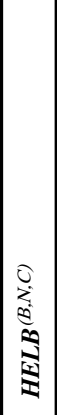 & 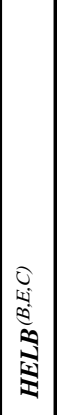 & 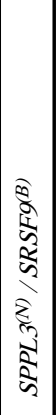 & 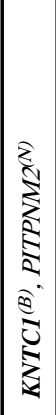 & $\mid$ & 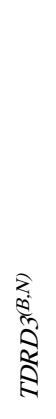 & 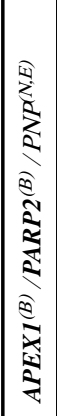 & 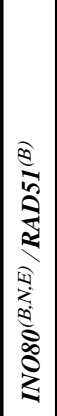 & $\left|\begin{array}{l}0 \\
0 \\
0 \\
5 \\
z \\
2 \\
5 \\
2 \\
2 \\
0 \\
0 \\
0 \\
0 \\
2\end{array}\right|$ & 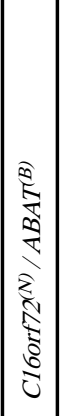 & 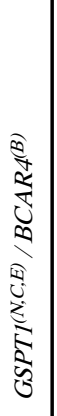 & 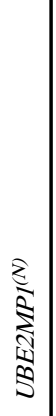 & $\begin{array}{l}\text { 孚 } \\
3 \\
3 \\
\frac{1}{2}\end{array}$ & 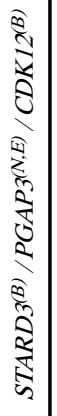 & 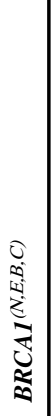 & 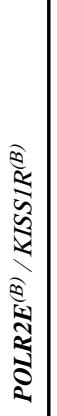 & 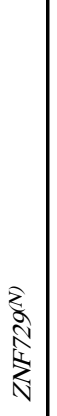 & 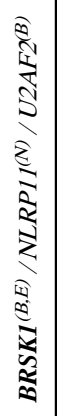 & 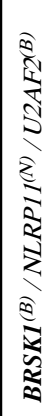 \\
\hline & $a$ & & 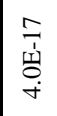 & 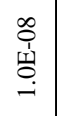 & & . & $\begin{array}{l}\vec{N} \\
\vec{\omega} \\
\stackrel{\omega}{-} \\
-\end{array}$ & 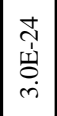 & 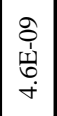 & . & & & & & & & & & , & & & & ' & ' & 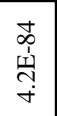 & $\begin{array}{l}\infty \\
0 \\
0 \\
\\
i\end{array}$ \\
\hline 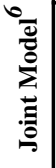 & 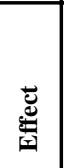 & & 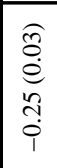 & 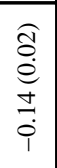 & & ' & 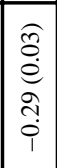 & $\begin{array}{c}\hat{\tilde{e}} \\
\stackrel{e}{e} \\
\bar{m} \\
\hat{i} \\
\end{array}$ & 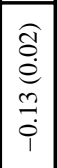 & .' & ' & & ' & & & I & 1 & ' & . & ' & & & ' & ' & 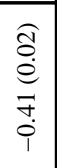 & 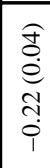 \\
\hline$\frac{\bar{s}}{8}$ & A & $\begin{array}{l}\infty \\
\text { o } \\
\text { I্. } \\
\dot{m}\end{array}$ & \begin{tabular}{l} 
\pm \\
\multirow{1}{1}{} \\
$\omega$ \\
$\infty$ \\
$\infty$
\end{tabular} & 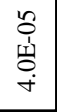 & $\begin{array}{l}\text { oे } \\
\text { ț } \\
\text { th } \\
\text { i }\end{array}$ & $\begin{array}{l}\stackrel{\oplus}{1} \\
\stackrel{5}{\infty} \\
\stackrel{-}{-}\end{array}$ & $\begin{array}{c}0 \\
o \\
1 \\
\text { wa } \\
\text { - } \\
\end{array}$ & 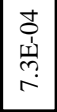 & \begin{tabular}{|l|}
0 \\
0 \\
1 \\
1 \\
6 \\
6 \\
\end{tabular} & $\begin{array}{l}\infty \\
0 \\
1 \\
\text { 岕 } \\
\dot{m} \\
\end{array}$ & 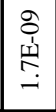 & 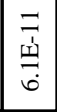 & $\begin{array}{l}0 \\
\frac{1}{1} \\
\text { wh } \\
\text { i }\end{array}$ & $\begin{array}{l}0 \\
\stackrel{1}{1} \\
\text { 岁 } \\
\text { i }\end{array}$ & 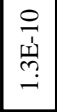 & $\underset{\stackrel{9}{\vec{t}}}{\stackrel{\overbrace{}}{+}}$ & \begin{tabular}{|c|c|}
$\infty$ \\
0 \\
w \\
$m$ \\
\end{tabular} & $\begin{array}{c}\stackrel{n}{7} \\
\stackrel{n}{m} \\
\stackrel{m}{-}\end{array}$ & $\begin{array}{l}\infty \\
0 \\
\stackrel{1}{1} \\
\stackrel{m}{m}\end{array}$ & $\begin{array}{l}\stackrel{8}{0} \\
\text { w్ } \\
\stackrel{n}{-}\end{array}$ & $\begin{array}{l}\text { oे } \\
\text { ज్ } \\
\stackrel{-}{-}\end{array}$ & 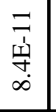 & $\begin{array}{l}\stackrel{ }{1} \\
\stackrel{+}{+} \\
\stackrel{-}{*}\end{array}$ & $\begin{array}{l}\infty \\
\stackrel{1}{1} \\
\text { 山ेt } \\
\stackrel{+}{+}\end{array}$ & $\begin{array}{l}\infty \\
\infty \\
w \\
w \\
w \\
n \\
n\end{array}$ & $\begin{array}{l}\frac{m}{1} \\
\dot{w} \\
\dot{w}\end{array}$ \\
\hline 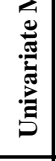 & 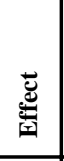 & $\begin{array}{l}\widehat{\delta} \\
\stackrel{\delta}{e} \\
= \\
\text { i }\end{array}$ & 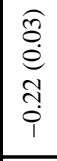 & $\begin{array}{l}\widehat{o} \\
\stackrel{0}{0} \\
0 \\
0 \\
0 \\
0\end{array}$ & 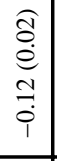 & $\begin{array}{c}\widehat{\hat{\theta}} \\
\stackrel{e}{0} \\
\bar{m} \\
i \\
i\end{array}$ & $\mid \begin{array}{l}\widehat{\sigma} \\
0 \\
\dot{0} \\
o \\
0 \\
i \\
i\end{array}$ & 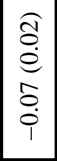 & 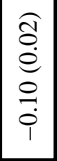 & $\begin{array}{c}\widehat{\widehat{c}} \\
\stackrel{0}{0} \\
m \\
\stackrel{+}{i}\end{array}$ & $\mid \begin{array}{c}\widehat{o} \\
\stackrel{0}{0} \\
\stackrel{9}{0} \\
\stackrel{1}{i}\end{array}$ & 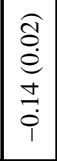 & $\begin{array}{l}\widehat{\widehat{c}} \\
\stackrel{0}{0} \\
\infty \\
\frac{\infty}{i} \\
\end{array}$ & $\mid \begin{array}{c}\widehat{\sigma} \\
0 \\
e \\
\pm \\
\vdots \\
i \\
i\end{array}$ & 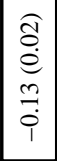 & $\begin{array}{c}\widehat{\delta} \\
\stackrel{0}{0} \\
\stackrel{+}{+} \\
\hat{1}\end{array}$ & 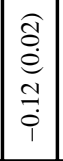 & $\begin{array}{c}\widehat{\widehat{c}} \\
\dot{0} \\
\stackrel{0}{0} \\
\stackrel{-}{0}\end{array}$ & 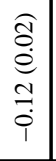 & 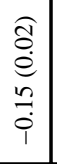 & $\begin{array}{l}\widehat{c} \\
\stackrel{0}{0} \\
\stackrel{0}{0} \\
\stackrel{1}{0}\end{array}$ & 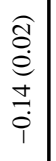 & 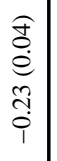 & $\begin{array}{c}\widehat{\widehat{D}} \\
\stackrel{0}{0} \\
= \\
\dot{1}\end{array}$ & $\begin{array}{l}\widehat{\widehat{c}} \\
\stackrel{0}{0} \\
\vec{j} \\
\dot{1}\end{array}$ & 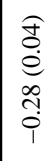 \\
\hline & $\mathbf{z}$ & స్ & 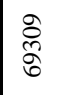 & $\begin{array}{l}\text { Oे } \\
\text { ठे }\end{array}$ & $\frac{7}{8}$ & $\begin{array}{l}0 \\
0 \\
0 \\
6\end{array}$ & $\frac{8}{8}$ & $\mid \begin{array}{l}\hat{N} \\
\tilde{D} \\
0\end{array}$ & 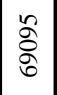 & $\begin{array}{l}\bar{\vdots} \\
\overline{0} \\
\end{array}$ & $\begin{array}{l}尺 \\
\infty \\
\infty \\
\infty \\
0\end{array}$ & $\begin{array}{l}\hat{y} \\
\text { 它 }\end{array}$ & $\frac{t}{\sigma}$ & $\begin{array}{l}\infty \\
\widetilde{N} \\
\infty \\
0\end{array}$ & $\frac{\bar{\sigma}}{\sigma}$ & $\begin{array}{l}\infty \\
0 \\
\tilde{b}^{\infty}\end{array}$ & 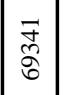 & $\begin{array}{l}0 \\
0 \\
0 \\
0 \\
0\end{array}$ & 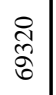 & $\begin{array}{l}n \\
\vec{r} \\
0 \\
0\end{array}$ & \begin{tabular}{l} 
ठे \\
\multirow{్}{0}{}
\end{tabular} & $\begin{array}{l}\text { సે } \\
\text { ठે }\end{array}$ & 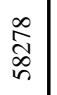 & 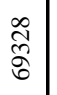 & ๙ે & 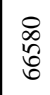 \\
\hline & 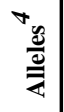 & $\begin{array}{l}\vec{b} \\
\stackrel{\dot{b}}{b} \\
\vec{a}\end{array}$ & 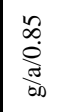 & 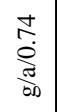 & $\begin{array}{c}\hat{f} \\
\stackrel{b}{\Delta} \\
\substack{a \\
\vec{a}}\end{array}$ & $\overrightarrow{\stackrel{p}{0}}$ & $\mid \begin{array}{l}\sqrt{2} \\
\tilde{2} \\
3 \\
3\end{array}$ & 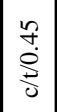 & 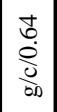 & 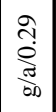 & $\mid \begin{array}{l}n \\
0 \\
0 \\
0.0 \\
0\end{array}$ & $\mid \begin{array}{c}m \\
\stackrel{q}{\rho} \\
\stackrel{g}{g} \\
\text { bo }\end{array}$ & $\begin{array}{l}\stackrel{\bullet}{0} \\
\stackrel{\leftrightarrow}{0} \\
\stackrel{0}{b 0}\end{array}$ & 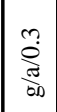 & 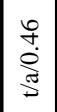 & 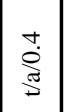 & $\mid \begin{array}{l}\infty \\
\stackrel{\infty}{0} \\
\stackrel{3}{0} \\
\end{array}$ & 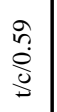 & $\begin{array}{l}\vec{n} \\
\stackrel{s}{s} \\
\stackrel{s}{s}\end{array}$ & $\begin{array}{l}\stackrel{2}{2} \\
\stackrel{\rho}{\circ} \\
\frac{b 0}{\sigma}\end{array}$ & 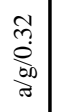 & 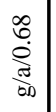 & $\begin{array}{l}\stackrel{m}{0} \\
\stackrel{0}{0} \\
\frac{5}{b 0}\end{array}$ & 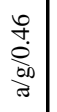 & 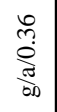 & 仓े \\
\hline & 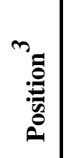 & $\begin{array}{l}0 \\
\infty \\
0 \\
0 \\
\infty \\
i\end{array}$ & 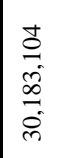 & 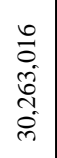 & 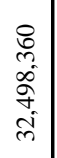 & 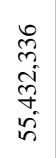 & $\begin{array}{c}\hat{E} \\
\hat{6} \\
\hat{D} \\
\approx \\
0 \\
\dot{0}\end{array}$ & 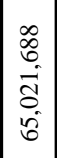 & 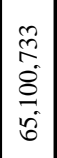 & $\begin{array}{l}0 \\
i \\
\sigma \\
\sigma \\
0 \\
\varrho \\
ٍ\end{array}$ & 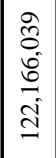 & 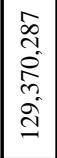 & 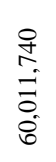 & 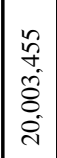 & 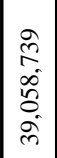 & \begin{tabular}{|c|}
0 \\
0 \\
0 \\
0 \\
0 \\
0 \\
0 \\
$\infty$ \\
$\infty$
\end{tabular} \mid & 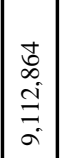 & 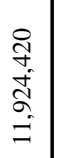 & 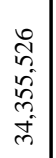 & 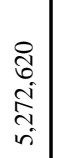 & 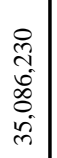 & $\begin{array}{l}\hat{\alpha} \\
\alpha \\
\infty \\
o \\
\sigma \\
\infty \\
\infty\end{array}$ & 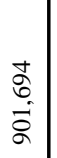 & 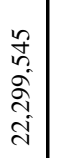 & 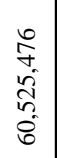 & $\begin{array}{l}0 \\
0 \\
0 \\
8 \\
8 \\
0 \\
0\end{array}$ \\
\hline & $\tilde{z}$ & 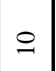 & $=$ & $=$ & $=$ & $\simeq$ & $\simeq$ & $\simeq$ & $\simeq$ & $\simeq$ & $\simeq$ & $\simeq$ & 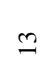 & \pm & $\cong$ & $\simeq$ & 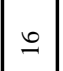 & $\stackrel{\imath}{ }$ & $\stackrel{0}{-1}$ & $=$ & $=$ & $=$ & 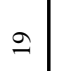 & 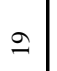 & 2 & 2 \\
\hline & 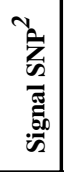 & 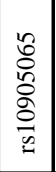 & $\frac{\stackrel{8}{0}}{\frac{0}{0}}$ & 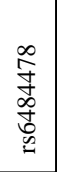 & 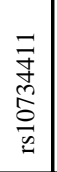 & 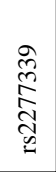 & 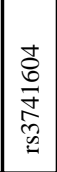 & 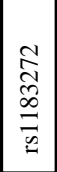 & \begin{tabular}{|l}
$\overrightarrow{0}$ \\
$\infty$ \\
0 \\
$\hat{0}$ \\
0
\end{tabular} & $\begin{array}{l}\infty \\
o \\
0 \\
w \\
0 \\
0\end{array}$ & 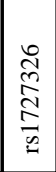 & 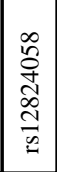 & 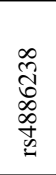 & 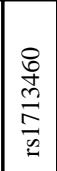 & $\mid \begin{array}{l}\circ \\
\circ \\
\vdots \\
0\end{array}$ & $\begin{array}{l}\frac{n}{c} \\
\infty \\
0 \\
\frac{0}{0} \\
\underline{n}\end{array}$ & 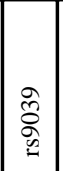 & 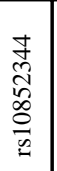 & 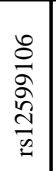 & 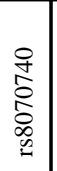 & 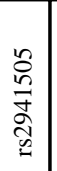 & 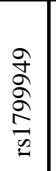 & 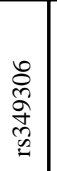 & $\begin{array}{l}\frac{0}{2} \\
\hat{2} \\
\hat{n} \\
\hat{n} \\
\hat{n}\end{array}$ & 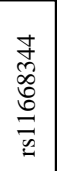 & 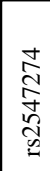 \\
\hline & 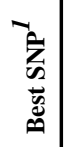 & 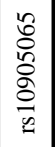 & 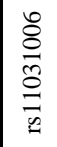 & : & 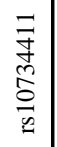 & 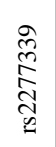 & 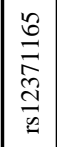 & : & 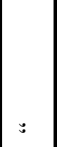 & $\begin{array}{l}\infty \\
\stackrel{0}{0} \\
\tilde{n} \\
0 \\
0\end{array}$ & 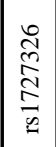 & 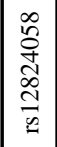 & 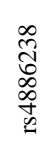 & 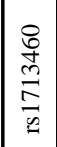 & 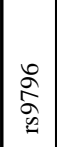 & 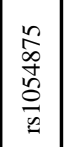 & 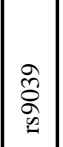 & 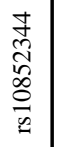 & 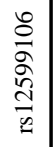 & 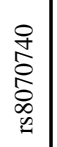 & 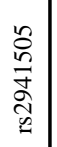 & 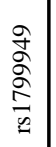 & 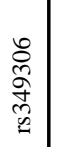 & 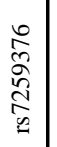 & 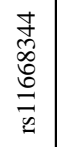 & \\
\hline & 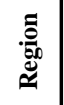 & & $\stackrel{* \frac{\pi}{\pi}}{\pi}$ & $\stackrel{*}{*} \stackrel{*}{\vec{N}}$ & $\mathcal{A}$ & * & 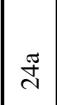 & 守 & 导 & $\ddot{\sim}$ & $\stackrel{2}{\sim}$ & $\hat{\imath}$ & " & ते & $\therefore$ & ${ }^{*} \vec{m}$ & ले & *m & मे & 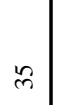 & $\stackrel{i}{i}$ & $\hat{m}$ & $\infty$ & के & * & 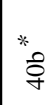 \\
\hline
\end{tabular}




\begin{tabular}{|c|c|c|c|c|c|c|c|c|}
\hline & 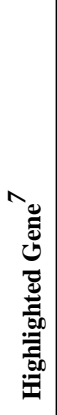 & 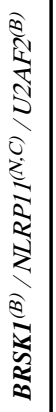 & 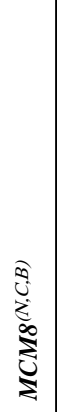 & 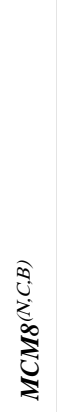 & 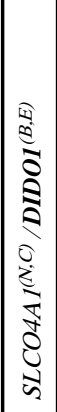 & 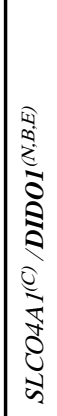 & (2) & 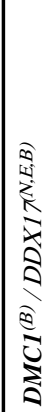 \\
\hline \multirow[b]{2}{*}{ 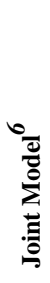 } & $a$ & 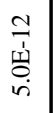 & 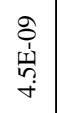 & 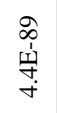 & 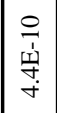 & 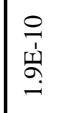 & & ' \\
\hline & 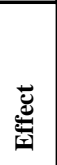 & $\begin{array}{c}\widehat{c} \\
\stackrel{0}{e} \\
\stackrel{0}{0} \\
0 \\
i\end{array}$ & $\begin{array}{l}\widehat{\widetilde{Q}} \\
\stackrel{0}{0} \\
\hat{i} \\
\hat{i}\end{array}$ & $\begin{array}{l}\hat{f} \\
\stackrel{0}{0} \\
\infty \\
\infty \\
\infty \\
i\end{array}$ & $\begin{array}{c}\widehat{\hat{0}} \\
\stackrel{0}{0} \\
0 \\
\dot{0} \\
1\end{array}$ & $\begin{array}{c}\widehat{\delta} \\
\tilde{O} \\
0 \\
0 \\
0 \\
0 \\
1\end{array}$ & & , \\
\hline \multirow{2}{*}{ 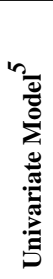 } & A & 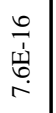 & $\begin{array}{l}\stackrel{\text { oे }}{ } \\
\text { I্t0 } \\
\stackrel{+}{+}\end{array}$ & $\begin{array}{l}\text { के } \\
\text { ț } \\
\stackrel{1}{-} \\
-\end{array}$ & $\mid \begin{array}{l}\frac{0}{1} \\
\underline{1} \\
6 \\
6\end{array}$ & 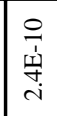 & 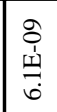 & $\begin{array}{l}m \\
\stackrel{m}{\omega} \\
\tilde{n} \\
\sim\end{array}$ \\
\hline & 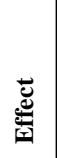 & 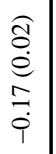 & $\begin{array}{l}\widehat{\sigma} \\
\stackrel{0}{0} \\
\stackrel{\leftrightarrow}{1} \\
i \\
i\end{array}$ & $\begin{array}{l}\hat{f} \\
\stackrel{0}{0} \\
\infty \\
\infty \\
\infty \\
i\end{array}$ & 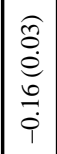 & $\begin{array}{c}\widehat{\delta} \\
\tilde{O} \\
0 \\
0 \\
0 \\
0 \\
1\end{array}$ & $\mid \begin{array}{c}\widehat{\sigma} \\
e \\
0 \\
0 \\
0 \\
0 \\
1\end{array}$ & $\mid \begin{array}{c}\widehat{\delta} \\
0 \\
0 \\
0 \\
0 \\
0 \\
1\end{array}$ \\
\hline \multirow{2}{*}{ 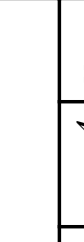 } & z & $\begin{array}{l}\infty \\
\vec{\infty} \\
0\end{array}$ & $\begin{array}{l}\text { वे } \\
\text { ț }\end{array}$ & 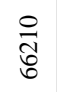 & 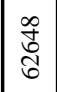 & $\overline{\tilde{\sigma}}$ & $\begin{array}{l}\tilde{\sigma} \\
\tilde{\sigma} \\
\text { a }\end{array}$ & $\begin{array}{l}\tilde{\delta} \\
\text { d. }\end{array}$ \\
\hline & $\begin{array}{l}\nabla_{\tilde{e}} \\
\frac{\underline{\underline{e}}}{\bar{E}}\end{array}$ & 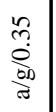 & 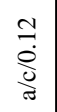 & $\begin{array}{l}\hat{\Omega} \\
\hat{\rho} \\
\frac{\hat{\rho}}{b 0}\end{array}$ & 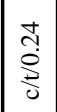 & 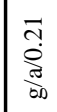 & 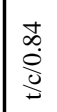 & 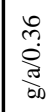 \\
\hline & 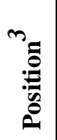 & $\begin{array}{l}n \\
a \\
i \\
0 \\
0 \\
0 \\
0\end{array}$ & $\begin{array}{l}\partial \\
\partial \\
\partial \\
\infty \\
\text { i. }\end{array}$ & $\begin{array}{l}\text { în } \\
\text { o. } \\
0 \\
\text { in }\end{array}$ & \begin{tabular}{|l|}
$\infty$ \\
$\infty$ \\
0 \\
0 \\
0 \\
0 \\
0
\end{tabular} \mid & 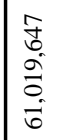 & $\mid \begin{array}{l}\vec{n} \\
\text { in } \\
\hat{\delta} \\
\text { co } \\
\text { in }\end{array}$ & 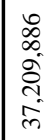 \\
\hline & $\tilde{z}$ & 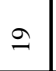 & ते & ते & ¿ & i & $\tilde{A}$ & ส \\
\hline & 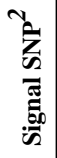 & 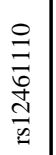 & 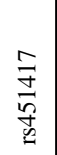 & $\begin{array}{l}\frac{n}{0} \\
\frac{0}{a} \\
\frac{6}{0} \\
0\end{array}$ & $\begin{array}{l}\tilde{R} \\
\hat{\delta} \\
\tilde{N} \\
\tilde{n}\end{array}$ & 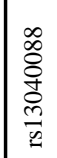 & 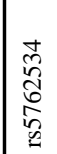 & 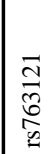 \\
\hline & 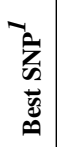 & : & $\begin{array}{l}\frac{n}{b} \\
\frac{a}{a} \\
\frac{b}{n}\end{array}$ & : & 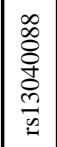 & = & 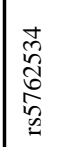 & \begin{tabular}{|l}
$\overline{\mathrm{s}}$ \\
$\vec{\delta}$ \\
$\underline{n}$
\end{tabular} \\
\hline & 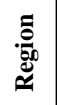 & $*^{*} \stackrel{\square}{q}$ & "* $\frac{\sigma}{\sigma}$ & $\stackrel{*}{\circ}$ & Эี & శి & $q$ & $\forall$ \\
\hline
\end{tabular}

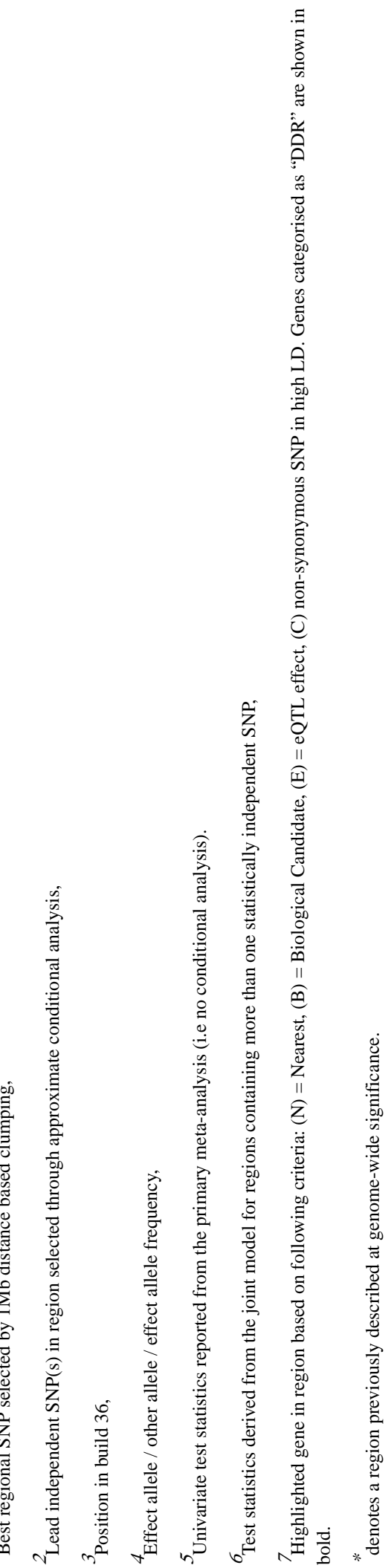




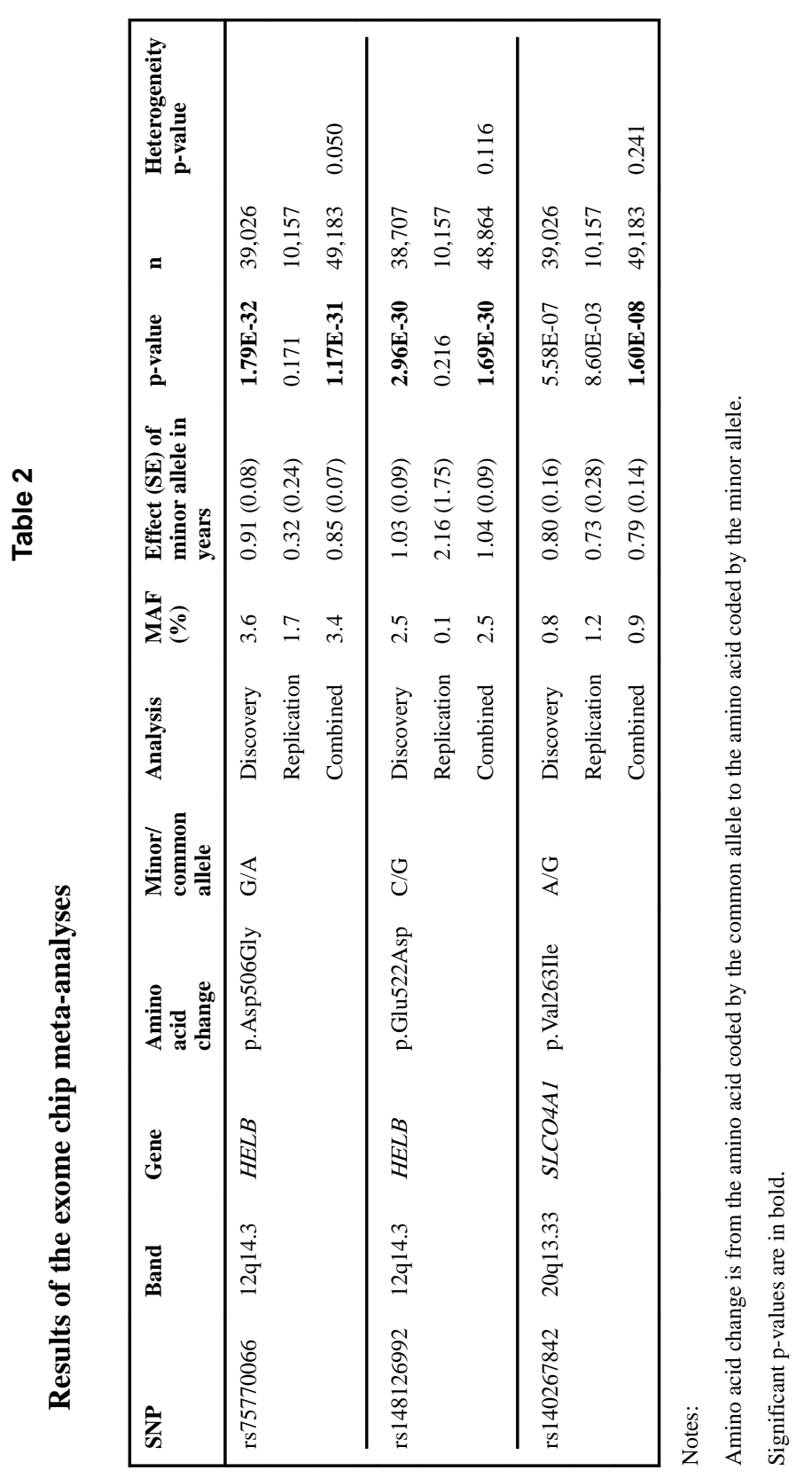

Nat Genet. Author manuscript; available in PMC 2016 May 01. 